Claremont Colleges

Scholarship@ Claremont

All HMC Faculty Publications and Research

HMC Faculty Scholarship

5-1-1996

\title{
The Interaction of Intense Laser Pulses with Atomic Clusters
}

T. Ditmire

Lawrence Livermore National Laboratory

Thomas D. Donnelly

Harvey Mudd College

A. M. Rubenchik

Lawrence Livermore National Laboratory

R. W. Falcone

University of California-Berkeley

M.D. Perry

Lawrence Livermore National Laboratory

\section{Recommended Citation}

T. Ditmire, T.D. Donnelly, A. M. Rubenchik, R.W. Falcone, M.D. Perry, The Interaction of Intense Laser Pulses with Atomic Clusters, Phys. Rev. A 53, 3379 (1996). doi: 10.1103/PhysRevA.53.3379

This Article is brought to you for free and open access by the HMC Faculty Scholarship at Scholarship @ Claremont. It has been accepted for inclusion in All HMC Faculty Publications and Research by an authorized administrator of Scholarship @ Claremont. For more information, please contact scholarship@cuc.claremont.edu. 


\title{
Interaction of intense laser pulses with atomic clusters
}

\author{
T. Ditmire, ${ }^{1, *}$ T. Donnelly, ${ }^{2}$ A. M. Rubenchik, ${ }^{1}$ R. W. Falcone,${ }^{2}$ and M. D. Perry ${ }^{1}$ \\ ${ }^{1}$ Laser Program, Lawrence Livermore National Laboratory, P.O. Box 808, L-443, Livermore, California 94550 \\ ${ }^{2}$ Department of Physics, University of California at Berkeley, Berkeley, California 94720
}

(Received 13 October 1995)

\begin{abstract}
We examine the interaction of intense, femtosecond laser radiation with the large (50-200 $⿱$ ) clusters produced in pulsed gas jets. Both experiment and simulation show that the plasmas produced during these interactions exhibit electron temperatures far in excess of that predicted by above-threshold ionization theory for a low-density gas. Efficient heating of the clusters by the laser is followed by rapid expansion of the clusters and long-lived x-ray emission from hot, decaying, underdense plasma. [S1050-2947(96)07005-9]
\end{abstract}

PACS number(s): $36.40 . \mathrm{Gk}, 52.50 . \mathrm{Jm}, 52.25 . \mathrm{Nr}$

\section{INTRODUCTION}

In recent years, much effort has gone into the understanding of the interaction of short $(\leqslant 1 \mathrm{ps})$, intense $\left(>10^{15}\right.$ $\mathrm{W} / \mathrm{cm}^{2}$ ) laser pulses with matter [1]. These experiments have typically involved studying the interaction of these highintensity laser pulses with either a low-density gas $\left(<10^{19}\right.$ atoms $\left./ \mathrm{cm}^{3}\right)$ or a high-density solid target $\left(\sim 10^{23}\right.$ atoms $\left./ \mathrm{cm}^{3}\right)$. Many of these studies have been motivated by a desire to generate photons and particles with energies far above the energy of a single laser photon. In particular, some of these experiments have investigated the production of incoherent and coherent $\mathrm{x}$-ray radiation or the generation of energetic electrons.

In gas targets, much progress has been made in the generation of bright, coherent soft $\mathrm{x}$ rays in the $30-100-\mathrm{eV}$ range through harmonic generation [2] and x-ray lasers $[3,4]$. Typically these experiments involve focusing the short laser pulse into a static filled gas cell or at the output of a pulsed gas jet. Gas phase targets are attractive for use in x-ray generation with short-pulse lasers for a number of reasons. Since the laser propagates through the medium, coherent $\mathrm{x}$-ray generation is possible through harmonic generation, or through the creation of a plasma channel suitable for x-ray laser gain. Furthermore, gas targets generate no debris. Gases, however, usually exhibit poor absorption of laser energy and are, therefore, limited in their effective use in converting laser light into $\mathrm{x}$ rays.

Solid targets, on the other hand, often exhibit very large laser energy absorption. The use of solids as targets for intense lasers has successfully produced both photons and particles with energies up to the $\mathrm{MeV}$ range $[5,6]$. Because of the strong absorption mechanisms in a solid density plasma, such as collisional heating, resonance absorption, and various plasma instabilities [7], significant fractions of the laser pulse energy $(>10 \%)$ can be deposited into the plasma $[8,9]$. The hot plasmas that result will emit copious amounts of $\mathrm{x}$ rays. Conversion efficiency of nearly $1 \%$ of laser light into $x$ rays in the $1-\mathrm{keV}$ photon energy range has been demonstrated $[10,11]$. This conversion efficiency can be increased

\footnotetext{
*Present address: Blackett Laboratory, Imperial College, London SW7 2BZ, United Kingdom.
}

to $10 \%$ when the surface of the target is coated with a layer of porous gold-black, which is composed of individual 100 - $\AA$ clusters of gold $[8,10]$. This gold-black was shown to be much more efficient at absorbing the incident laser energy than conventional flat gold targets due to the large surface to volume ratio of the gold clusters. Strong soft-x-ray yields have also been obtained when $\mathrm{C}_{60}$ molecules are illuminated with a short-pulse $\mathrm{KrF}$ laser [12], suggesting that the large laser energy absorption observed with gold-black targets also occurs when large molecules are the target media.

High-pressure gas jets produce a unique combination of both gas and solid phase components. Solid density clusters form in the jet, resulting from the cooling associated with the adiabatic expansion of the gas into vacuum [13]. This cooling causes the gas to supersaturate and nucleate. Under appropriate conditions, when the gas jet backing pressure exceeds a few atmospheres, the clusters formed in the expanding jet can be quite large $\left(>10^{4}\right.$ atoms per cluster) for gases such as $\mathrm{Ar}, \mathrm{Kr}, \mathrm{N}_{2}$, and $\mathrm{Xe}$.

Previously, McPherson et al. observed anomalous X-ray line emission from high $\mathrm{Kr}$ and $\mathrm{Xe}$ charge states when a high-intensity $\left(0.5-80 \times 10^{17} \mathrm{~W} / \mathrm{cm}^{2}\right), 248-\mathrm{nm}$ laser was focused into a jet of these gases $[14,15]$. It was suggested that this emission was the result of inner shell vacancies produced by collisions of laser driven electrons with atoms in small $(\sim 2-100$ atom) clusters, and that the observed emission was prompt $(\sim 100-1000 \mathrm{fs})$ after excitation by the laser. We have observed similar strong x-ray emission from a gas jet containing clusters, but we find that the emission from these interactions is long lived ( $\sim 1 \mathrm{~ns})$, contrary to the hypothesis of McPherson et al. [16]. In our experiments the vast majority of the $\mathrm{x}$ rays emitted from the laser-cluster interaction does not come from the clusters themselves, but we find that the clusters serve only to absorb the laser energy, with the bulk of the x-ray emission occurring after the cluster has expanded.

In this paper we examine the interaction of femtosecond laser pulses with large clusters in detail. We find that the atomic clusters are very efficient at absorbing laser energy, with the coupling of the laser energy into the cluster being predominantly by collisional heating of electrons. Large heating within the cluster results in rapid production of high ion charge states, followed by long-lived $(\sim \mathrm{ns}) \mathrm{x}$-ray emission from the hot, underdense plasma that results after the 
clusters have expanded. We present experimental as well as computational results on this interaction and find good agreement between experiments and simulations.

\section{CLUSTER FORMATION IN A GAS JET}

When high-pressure gas flows into vacuum through an orifice, the expansion is isentropic; the random thermal energy is converted into directed kinetic energy resulting in a decrease in temperature. For certain gases, when the backing pressure is high ( $>10$ psi for typical gas jets), the gas can become supersaturated and solid density droplets will form [13]. These droplets, which are held together by van der Waals forces, can become quite large under appropriate conditions. Very extensive studies of these processes have been carried out and these allow accurate predictions of the threshold and size of clusters that can be produced under a variety of experimental conditions [13,17-19].

Hagena and Obert found that the onset of clustering in gas jets and the size variation of the clusters produced can be characterized by an empirical parameter that varies with the experimental conditions of the gas jet $[13,18]$. This Hagena parameter is given by

$$
\Gamma^{*}=k \frac{(d / \tan \alpha)^{0.85} p_{0}}{T_{0}^{2.29}}
$$

where $d$ is the jet throat diameter in $\mu \mathrm{m}, \alpha$ is the jet expansion half angle $\left(=45^{\circ}\right.$ for a sonic expansion, but smaller for a supersonic expansion), $p_{0}$ is the backing pressure in mbar, $T_{0}$ is the initial gas temperature, and $k$ is a constant that depends on the atomic species $(k \approx 2900$ for $\mathrm{Kr}, 1700$ for $\mathrm{Ar}$, 180 for $\mathrm{Ne}$, and 4 for $\mathrm{He}$ [20]). Most studies have shown that clustering begins when this parameter exceeds $\sim 300$ with the average size of clusters produced increasing roughly as $\sim\left(\Gamma^{*}\right)^{2.0-2.5}[13,21]$. These studies show that large clusters $\left(>10^{4}\right.$ atoms/cluster) predominate when $\Gamma^{*}>5 \times 10^{4}$ [20]. This scaling indicates, first, that clustering is more significant for the heavier noble gases such as $\mathrm{Kr}$ and $\mathrm{Ar}$ than for $\mathrm{Ne}$ and He. For a typical gas jet throat of $\sim 1 \mathrm{~mm}$, backing pressures as low as $\sim 300 \mathrm{psi}$ are sufficient to produce large clusters at room temperature in $\mathrm{Kr}$ or $\mathrm{Ar}$, while $>2000$ psi is required for the onset of any clustering at all in helium. The scaling of Eq. (1) also suggests that clustering is enhanced when the jet flow expansion angle is restricted by a shaped nozzle downstream from the jet throat. The increase of clustering with the use of a supersonic nozzle has been confirmed for a wide variety of gases [22].

Though small clusters of $\mathrm{Ne}$ and He have been observed under certain conditions [13], the scaling of Eq. (1) suggests that $\mathrm{Ar}, \mathrm{Kr}, \mathrm{N}_{2}$, and $\mathrm{Xe}$ are most favorable for the production of large clusters. We can estimate the size of clusters that will be desirable for illumination by a short-pulse laser, if we require that the time it takes for the cluster to disassemble is comparable to or longer than the laser pulse. As will be discussed in Sec. IV C, the predominant expansion mechanism for the clusters of interest is hydrodynamic expansion of the cluster plasma sphere after the electrons have been heated by the laser pulse. We can estimate the time of this expansion if we assume sonic expansion and require the den- sity to drop from the solid cluster density to the surrounding ambient gas density. The resulting cluster expansion time is approximately

$$
\tau_{\mathrm{ex}} \approx r_{0}\left(\frac{m_{i}}{Z k T_{e}}\right)^{1 / 2}\left(\frac{n_{0}}{n_{e}}\right)^{1 / 3},
$$

where $r_{0}$ is the initial cluster radius, $k T_{e}$ is the cluster electron temperature, and $m_{i}$ is the ion mass. For an argon cluster (which has an initial lattice spacing of approximately $3.8 \AA$ [23]) with an initial electron temperature of $1 \mathrm{keV}$, a $Z \approx 8$, and a surrounding bulk plasma with a density of $10^{18}$ atoms $/ \mathrm{cm}^{3}$, the expansion time will be longer than approximately 1 ps if the initial cluster is larger than about $100 \AA$ in diameter. This corresponds to about $10^{5}$ atoms per cluster. We expect, therefore, that 1-0.1-ps laser pulses will interact with the clusters over the majority of the laser pulse if the clusters are of the order of $100 \AA$. Pulses much longer than $\sim 1 \mathrm{ps}$, however, will only interact with the cluster during the leading edge of the pulse.

Illumination of a gas jet producing large clusters with a sufficiently short pulse will, therefore, deposit laser energy in a manner that is quite different than a low-density gas without clusters. Most importantly, the electron-ion collision frequency of the plasma produced is greatly enhanced by the large local density inside the cluster. Significant electron heating during the laser pulse will result. This is in contrast to a low-density gas, on the other hand, which will tend to exhibit low electron temperatures $(<50 \mathrm{eV})$ since efficient heating mechanisms are absent.

\section{INTERACTION OF A SHORT-PULSE LASER WITH A LOW-DENSITY GAS WITHOUT CLUSTERS}

The interaction of an intense laser pulse with a lowdensity gas and the characteristics of the plasmas that result have been extensively studied in recent years. These studies have been largely motivated by $\mathrm{x}$-ray laser development $[24,3,4]$. When the density of the atoms is low enough that collisions between electrons and ions is infrequent on the time scale of the pulse, the dominant mechanism for plasma production is by direct optical ionization of the atoms by the laser field. For the intensities of interest in these studies, the ionization is primarily through tunnel ionization of the electrons [25]. In a plasma formed by tunnel ionization there are three primary mechanisms for heating the plasmas that are produced in the gas.

The first heating mechanism is above-threshold ionization (ATI). This heating is a single atom effect and results from the additional energy that the electron receives from the laser field during the ionization process. Burnett and Corkum have derived an expression for the heating of optically ionized electrons based on a classical analysis of the electron trajectory in the laser field after tunneling [24]. In this model, the residual electron energy is determined by the phase at which the electron is ionized in the oscillating laser field. The residual drift energy acquired by the electron in this approximation is simply

$$
E_{\mathrm{ATI}}=2 U_{p} \sin \Delta \phi,
$$




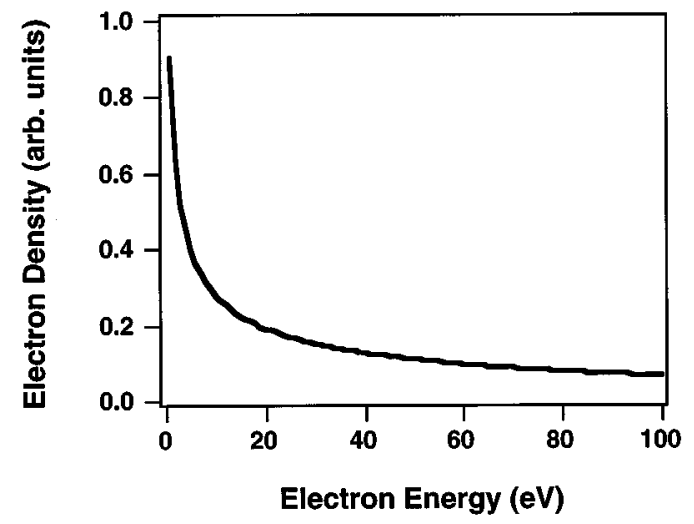

FIG. 1. Energy distribution of helium atoms ionized by an $800-$ $\mathrm{nm}, 100$-fs pulse with a peak intensity of $10^{17} \mathrm{~W} / \mathrm{cm}^{2}$ calculated using the model of Burnett and Corkum.

where $U_{p}$ is the ponderomotive potential at the time that the electron is ionized, given by $U_{p}(\mathrm{eV})=9.3$ $\times 10^{-14}\left[I\left(\mathrm{~W} / \mathrm{cm}^{2}\right)\right]\left[\lambda^{2}(\mu \mathrm{m})\right]$ and $\Delta \phi$ is the phase off of the peak of the laser electric field at which the electron is ionized. Using this formula, it is straightforward to estimate the energy distribution function of electrons ionized by tunneling. For illustrative purposes the energy distribution of helium atoms subject to an $800-n m, 100$-fs pulse with a peak intensity of $10^{17} \mathrm{~W} / \mathrm{cm}^{2}$ calculated using the model of Burnett and Corkum is shown in Fig. 1. The distribution is very non-Maxwellian, strongly peaked at low energy. The tail of the distribution extends to a maximum energy of $2 U_{p}$. Since the distribution is strongly peaked around zero, the "temperature" of this distribution [defined as $k T_{e}=(2 / 3)\langle E\rangle_{\mathrm{avg}}$ ], however, is cold, equal to about $40 \mathrm{eV}$. Figure 2 shows the calculated temperature of a plasma formed from ionization of $\mathrm{He}, \mathrm{Ar}$, and $\mathrm{Kr}$, as a function of peak intensity for a $100-\mathrm{fs}$

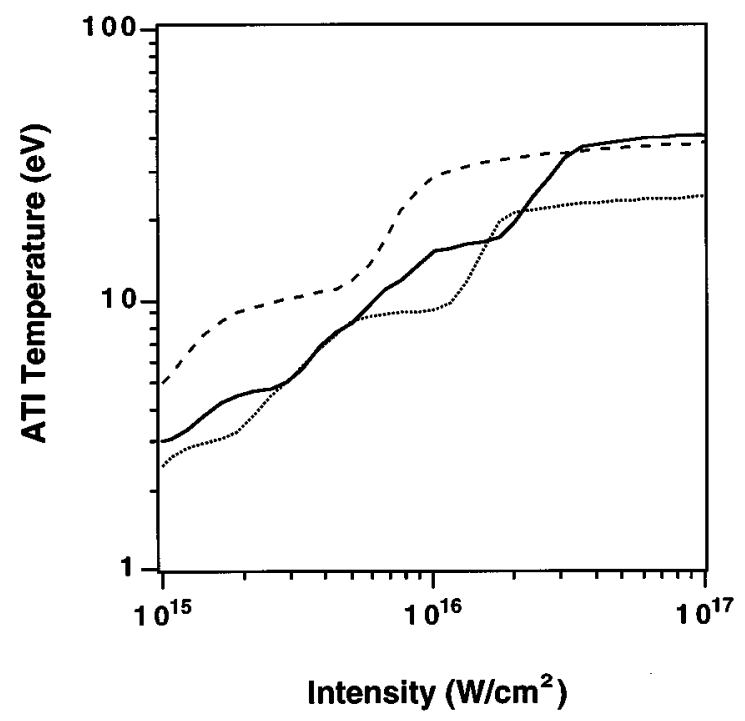

FIG. 2. Calculated temperature of a plasma formed from ionization of $\mathrm{He}$ (dashed line), $\mathrm{Ar}$ (solid line), and $\mathrm{Kr}$ (dotted line), as a function of peak intensity for a 100-fs (full width at half maximum), 800-nm linearly polarized laser pulse.

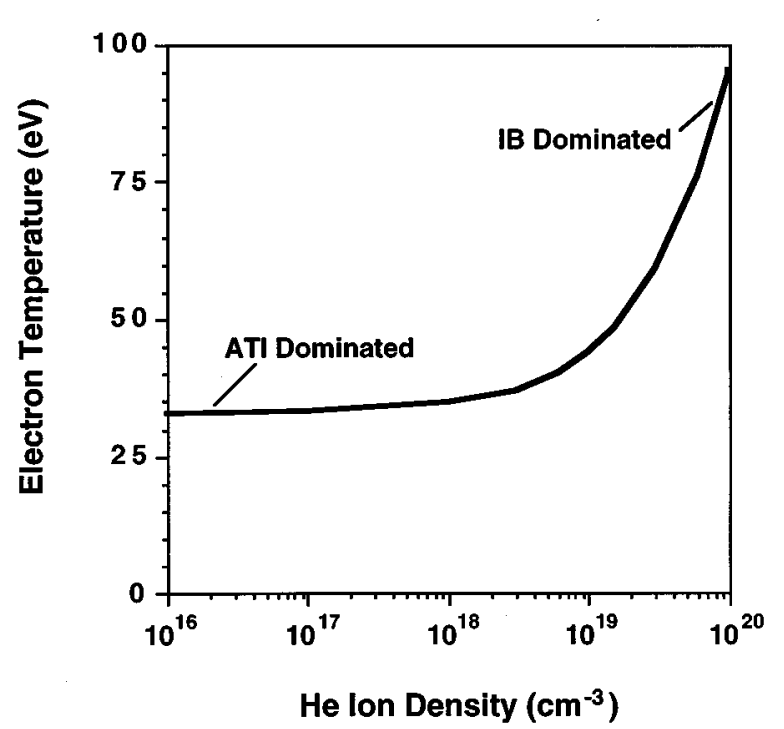

FIG. 3. Calculated temperature of a helium plasma as a function of density when illuminated by a 100 -fs, 800 -nm pulse with a peak intensity of $10^{16} \mathrm{~W} / \mathrm{cm}^{2}$ including the effects of ATI heating and collisional inverse bremsstrahlung.

(full width at half maximum), 800-nm linearly polarized pulse, based on the model of Burnett and Corkum and a numerical integration of the dc tunneling rate [25]. The steps in the temperature correspond to the intensities at which additional electrons are stripped from the ion. In general, the plasma temperature does not exceed $50 \mathrm{eV}$ in any of these gases for intensities below $\sim 10^{17} \mathrm{~W} / \mathrm{cm}^{2}$. The ATI prediction of a cold $(<50 \mathrm{eV})$ electron temperature in an optically ionized, low-density plasma has been confirmed experimentally [26].

Another potential mechanism for heating of electrons is through Coulomb collisions of laser driven electrons with the ions in the gas (or inverse bremsstrahlung). For typical gas densities, this mechanism is much less important than ATI heating [27]. The rate of energy deposition per electron can be written as

$$
\frac{\partial E_{\mathrm{coll}}}{\partial t}=\nu_{e i} U_{p}
$$

where $\nu_{e i}$ is the electron-ion collision frequency [defined in the strong-field and weak-field limits in Eqs. (20) and (21)]. Use of the strong-field limit for this collisional frequency, Eq. (20), predicts that for a $10-\mathrm{eV}$ He plasma, with an ion density of $10^{18} \mathrm{~cm}^{-3}$ at an intensity of $10^{16} \mathrm{~W} / \mathrm{cm}^{2}$, the heating rate is approximately $3 \mathrm{eV} / \mathrm{ps}$. So the contribution to the heating of gases is, in general, quite small for a subpicosecond laser pulse. To more accurately calculate the effects of collisional heating in gases, we have developed a code in which ATI heating based on the semiclassical model is included with collisional heating, which is calculated by using a Monte Carlo algorithm (described elsewhere [28]). The temperature of a helium plasma as a function of gas density when illuminated by a $100-\mathrm{fs}, 800-\mathrm{nm}$ pulse with a peak intensity of $10^{16} \mathrm{~W} / \mathrm{cm}^{2}$ is shown in Fig. 3. At the lowest densities the average temperature is simply determined by 
the ATI heating, $\approx 35 \mathrm{eV}$. Only when the ion density approaches $10^{20} \mathrm{~cm}^{-3}$ does the temperature begin to rise due to the collisional heating.

The third potential heating mechanism in an underdense plasma comes from stimulated Raman scattering (SRS) [7]. Wilks et al. have shown that heating from SRS is negligible if the product of the electron plasma wave growth rate $\gamma$, and the laser pulse duration $\tau$ is kept below 18-20 [29]. The plasma growth rate is [7]

$$
\gamma=\frac{v_{\mathrm{osc}} \omega}{2 c}\left(\frac{\omega_{p}}{\omega\left(1-\omega_{p} / \omega\right)}\right)^{1 / 2},
$$

where $\omega_{p}$ is the plasma frequency, and $v_{\mathrm{osc}}$ is the electron oscillation velocity in the laser field. For an 800-nm, 100-fs pulse propagating in an electron density of $10^{19} \mathrm{~cm}^{-3}$, this condition implies that SRS heating is not significant for intensities $<6 \times 10^{17} \mathrm{~W} / \mathrm{cm}^{2}$. This is consistent with the work of Crane et al. [30] and Blyth et al. [31] in which SRS was shown to be an important heating mechanism for laser intensities approaching $10^{18} \mathrm{~W} / \mathrm{cm}^{2}$, but not important at lower intensities.

In general, the temperatures of low-density gases illuminated by $100-\mathrm{fs}$ pulses at intensities below $10^{17} \mathrm{~W} / \mathrm{cm}^{2}$ are usually in the 10-50-eV range for the gases of interest. Only when high-density clusters are present in the gas will the absorption deviate significantly from this picture. When clusters are present in the gas, collisional processes, such as inverse bremsstrahlung and collisional ionization, will become dominant because of the much higher density within the cluster.

\section{THE THEORY OF SHORT-PULSE LASER INTERACTIONS WITH CLUSTERS}

To make quantitative predictions about the nature of intense laser pulse interactions with clusters we require a model that accounts for the various processes that occur on the time scale of the laser pulse. These will include laser energy absorption, ionization, and cluster expansion. To model these interactions, we can take advantage of the large size of the clusters considered in this work. We treat the interaction of the laser pulse with the cluster as that of the laser interacting with a small ball of high-density plasma.

We shall assume that the collisionality inside the cluster is high enough and the spatial size of the cluster is small enough that no thermal gradients exist within the cluster. Thus we can assume that the temperature is uniform and the electron velocity distribution is isotropic and described by a Maxwellian. This will be true when the electron thermalization time is small on the time scale of the laser interaction. Strictly speaking, this picture of the cluster as a small plasma ball is only valid when the cluster dimension is significantly larger than a Debye length $\lambda_{d}=\sqrt{k T_{e} / 4 \pi n_{e} e^{2}}$, which is equal to $\sim 5 \AA$ for a solid density, $1000-\mathrm{eV}$ plasma. Therefore it is a good description for the large clusters $(\sim 100 \AA)$ considered here.

We shall also assume that the density within the cluster is uniform throughout the cluster expansion. The ionization rate will be a combination of field ionization and collisional ionization rates. The heating will be predominantly by colli- sions (inverse bremsstrahlung). The expansion of the cluster will be by a combination of hydrodynamic expansion and a Coulomb explosion that results when a significant charge has accumulated on the cluster. This second mechanism requires a knowledge of the rate at which electrons "free stream" out of the cluster and to what degree they are retained by space charge. Before calculating this free streaming or the heating rate, we require knowledge of the ionization rate to determine the electron density in the cluster.

\section{A. Ionization mechanisms}

There are three ionization mechanisms which can be important in the interaction of the laser with the atoms of the cluster. The first mechanism is direct optical ionization of the atoms. This mechanism is most important in the early stages of the laser pulse interaction with the cluster since it produces the initial electrons that form the plasma. This rate can be calculated using the cycle-averaged tunnel ionization rate of Ammosov, Delone, and Krainov [25]. For an arbitratry atom this rate is given by

$$
\begin{aligned}
W_{\mathrm{tun}}= & \omega_{a} \frac{(2 l+1)(l+|m|) !}{2^{|m|}|m| !(l+|m|) !}\left(\frac{2 e}{n^{*}}\right)^{n^{*}} \frac{1}{2 \pi n^{*}} \\
& \times I_{p}\left(\frac{2 E}{\pi\left(2 I_{p}\right)^{3 / 2}}\right)^{1 / 2}\left(\frac{2\left(2 I_{p}\right)^{3 / 2}}{E}\right)^{2 n^{*}-|m|-1} \\
& \times \exp \left[\frac{2\left(2 I_{p}\right)^{3 / 2}}{3 E}\right]
\end{aligned}
$$

Here, $l$ and $m$ are the angular momentum quantum numbers (the rate is averaged over $m$ for a shell), $\omega_{a}$ is the atomic frequency $\left(\omega_{a}=4.13 \times 10^{16} \mathrm{~s}^{-1}\right), n^{*}$ is the effective principal quantum number, $n^{*}=Z\left[2 I_{p}(\mathrm{eV})\right]^{-1 / 2}, E$ is the field of the laser in atomic units, and $I_{p}$ is the ionization potential of the charge state in atomic units. This rate is very nonlinear with laser field. For subpicosecond pulses, a laser intensity of $>10^{14} \mathrm{~W} / \mathrm{cm}^{2}$ is typically required before tunnel ionization becomes significant. For example, nearly $100 \%$ ionization of neutral Ar in a 100-fs pulse occurs when the peak laser intensity reaches $\sim 3 \times 10^{14} \mathrm{~W} / \mathrm{cm}^{2}$.

The second ionization mechanism in the cluster turns out to be the most important for conditions of interest. This is ionization that occurs from inelastic collisions between electrons and ions. Once a few electrons are produced by optical ionization the production of higher charge states is dominated by collisional ionization as a result of the high density in the cluster. To calculate this rate in our model we use the empirically determined collisional ionization rate formula of Lotz [32]. This rate per ion averaged over a Maxwellian electron distribution is

$$
W_{k T}=n_{e} \frac{a_{i} q_{i}}{I_{p}\left(k T_{e}\right)^{1 / 2}} \int_{I_{p} / k T_{e}}^{\infty} \frac{e^{-x}}{x} d x
$$

where $n_{e}$ is the electron density, $I_{p}$ is the ionization potential in $\mathrm{eV}, a_{i}$ is an empirical constant equal to $4.5 \times 10^{-14} \mathrm{eV}^{2}$ $\mathrm{cm}^{-3}$ [32], and $q_{i}$ is the number of electrons in the outer shell of the ion. For the conditions that prevail in the cluster, this rate can be quite high. For example, in a solid density 
cluster with eight times ionized $\operatorname{Ar}(Z=8), n_{e}=2 \times 10^{23} \mathrm{~cm}^{-3}$ so at a temperature of $1 \mathrm{keV}$ the rate for ionization of the argon to $\mathrm{Ar}^{9+}$ from $\mathrm{Ar}^{8+}\left(I_{p}=422 \mathrm{eV}\right)$ is $0.3 \mathrm{fs}^{-1}$. Therefore this ionization mechanism can be responsible for the production of high charge states well below the intensity threshold for tunnel ionization in the cluster if sufficient heating occurs inside the cluster. (Note, for example, that the tunnel ionization mechanism to $\mathrm{Ar}^{9+}$ only becomes significant when the laser intensity reaches $>10^{18} \mathrm{~W} / \mathrm{cm}^{2}$.)

The ionization rate in Eq. (7) accounts only for the collisional ionization by electrons with thermal energy sufficient to ionize the ion. The electrons in the cluster also have a velocity associated with their oscillations in the field. In general, the rate of collisional ionization by electrons driven by a laser in a dense plasma is a difficult problem because of the large number of Coulomb collisions that the electrons undergo which randomizes their sinusoidal oscillation velocity. Nonetheless, we can estimate the magnitude of this ionization mechanism rate by considering electrons with pure sinusoidal velocity. This treatment will, in general, overestimate the laser driven electron collisional ionization rate since we allow the electrons to have larger peak oscillation velocities than they will really acquire due to randomization of the velocity by collisions.

To calculate this rate we use the collision ionization cross section of Lotz. The ionization rate per ion is

$$
W_{\text {las }}(t)=n_{e} \sigma_{i} \frac{e E}{m_{e} \omega}|\sin \omega t|,
$$

where $\sigma_{i}$ is the velocity-dependent cross section for ionization. It is convenient to determine the cycle-averaged rate of ionization for comparison to the other rates, Eqs. (6) and (7). We find this by averaging Eq. (8) over one laser cycle. The rate in Eq. (8) is nonzero only over that part of the laser cycle in which the driven electron has enough kinetic energy $K_{e}$ to ionize the ion (i.e., $K_{e}>I_{p}$ ). The cycle-averaged rate is

$$
W_{\mathrm{las}}=\left\langle W_{\mathrm{las}}(t)\right\rangle=\frac{n_{e}}{\pi} \int_{\phi_{\min }}^{\pi} \sigma_{i} \frac{e E}{m_{e} \omega} \sin \phi d \phi,
$$

where $\phi_{\min }$ is the phase at which the oscillating electron has the minimum amount of energy necessary to ionize the ion $\left(=I_{p}\right)$. The Lotz cross section for the ionization is [32]

$$
\sigma_{i}=a_{i} q_{i} \frac{\ln \left(K_{e} / I_{p}\right)}{K_{e} I_{p}}
$$

Using the fact that $\sin \phi d \phi=\left(4 U_{p}\right)^{-1}\left(1-K_{e} / 2 U_{p}\right)^{-1 / 2} d K_{e}$ (which follows from the expression for the kinetic energy, $K_{e}=2 U_{p} \sin ^{2} \phi$ ), the cycle-averaged ionization rate can be written

$$
\begin{aligned}
W_{\text {las }}= & n_{e} \frac{a_{i} q_{i}}{\pi I_{p}}\left(\frac{1}{m_{e} U_{p}}\right)^{1 / 2} \int_{l_{p}}^{2 U_{p}} \frac{\ln \left(K_{e} / I_{p}\right)}{2 K_{e}} \\
& \times \frac{1}{\sqrt{1-K_{e} / 2 U_{p}}} d K_{e} .
\end{aligned}
$$

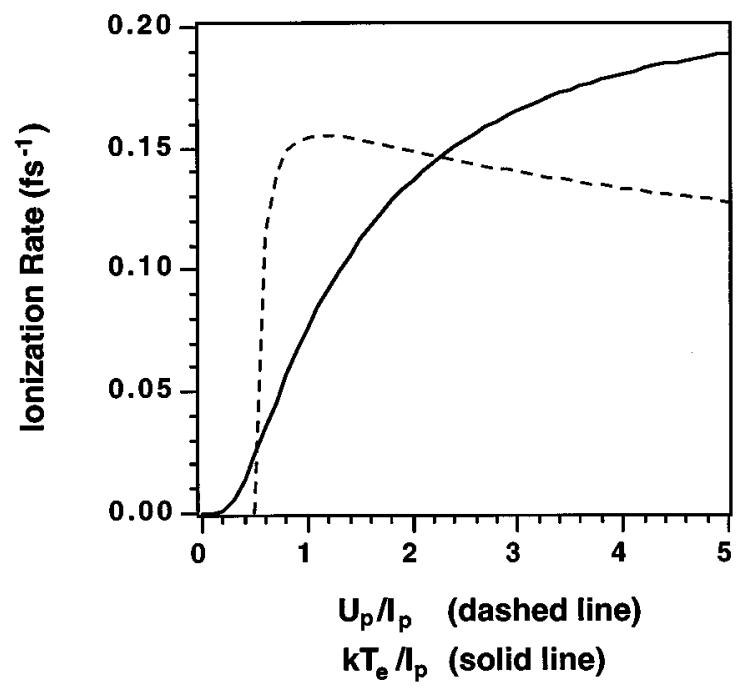

FIG. 4. Calculated ionization rate for $\mathrm{Ar}^{8+}$ ions in a plasma with an electron density of $10^{23} \mathrm{~cm}^{-3}$ from thermal electrons (solid line) and laser driven electrons (dashed line).

Equation (11) cannot be integrated directly but if we concern ourselves with the electrons of energies around the ionization potential where the ionization rate is highest we can expand the logarithm around the small parameter $1-I_{p} / K_{e}$ and write

$$
\ln \left(K_{e} / I_{p}\right) \approx \frac{3}{2}-2 \frac{I_{p}}{K_{e}}+\frac{I_{p}^{2}}{2 K_{e}^{2}} .
$$

This approximation turns out to be a good one not only for electron kinetic energies close to $I_{p}$ but for energies somewhat above $I_{p}$ as well (up to about $K_{e} \sim 2 I_{p}$ ). With this approximation, the cycle-averaged laser driven collisional ionization rate then becomes

$$
\begin{aligned}
W_{\text {las }} \approx & n_{e} \frac{a_{i} q_{i}}{2 \pi I_{p} m_{e}^{1 / 2} U_{p}^{1 / 2}}\left\{\left[3+\frac{I_{p}}{U_{p}}+\frac{3}{32}\left(\frac{I_{p}}{U_{p}}\right)^{2}\right]\right. \\
& \times \ln \left[\frac{1+\sqrt{1-I_{p} / 2 U_{p}}}{1-\sqrt{1-I_{p} / 2 U_{p}}}\right]-\left(\frac{7}{2}+\frac{3 I_{p}}{8 U_{p}}\right) \\
& \left.\times \sqrt{1-I_{p} / 2 U_{p}}\right\} .
\end{aligned}
$$

This ionization rate for $\mathrm{Ar}^{8+}$ is plotted in Fig. 4 as a function of $U_{p} / I_{p}$ for an electron density of $1 \times 10^{23} \mathrm{~cm}^{-3}$. The ionization rate is compared with the thermal ionization rate of Eq. (7) as a function of $k T_{e} / I_{p}$. From this comparison we see that these two rates are of comparable magnitude if the temperature of the plasma is comparable to the ponderomotive energy of the laser field inside the cluster. To accurately calculate the thermal ionization rate, however, we require knowledge of the electron temperature. We therefore need to examine the collisional heating mechanisms in the cluster.

\section{B. Cluster heating mechanisms}

Since we assume no temperature gradients in the cluster we can treat the heating from the laser as uniform over the 
cluster volume. We shall assume that the laser primarily deposits its energy into the free electrons in the cluster and that this energy deposition is through collisional inverse bremsstrahlung. The heating rate can be found by considering the laser energy deposition rate in a dielectric sphere. The energy deposited per unit volume in the cluster is therefore given by [33]

$$
\frac{\partial U}{\partial t}=\frac{1}{4 \pi} \mathbf{E} \cdot \frac{\partial \mathbf{D}}{\partial t}
$$

The laser field inside the cluster is $\mathbf{E}=\frac{1}{2} \hat{\mathbf{x}}\left(E e^{i \omega t}+\right.$ c.c. $)$ and $\mathbf{D}=\varepsilon \mathbf{E}$. The heating rate then becomes, upon time averaging over a laser cycle,

$$
\frac{\partial U}{\partial t}=\frac{\omega}{8 \pi} \operatorname{Im}[\varepsilon]|E|^{2} .
$$

Since the diameters of the clusters considered in our study are, in general, much smaller than the wavelength of the laser light, we can approximate the field inside the cluster by assuming that it is given by the solution for the field inside a dielectric sphere surrounded by a constant field. The solution for the field inside of a uniform sphere is [34]

$$
E=\frac{3}{|\varepsilon+2|} E_{0}
$$

where $E_{0}$ is the strength of the laser field in vacuum, outside the cluster. The heating rate inside the cluster becomes

$$
\frac{\partial U}{\partial t}=\frac{9 \omega}{8 \pi} \frac{\operatorname{Im}[\varepsilon]}{|\varepsilon+2|^{2}}\left|E_{0}\right|^{2} .
$$

We use a simple Drude model for the plasma dielectric constant:

$$
\varepsilon=1-\frac{\omega_{p}^{2}}{\omega(\omega+i \nu)}
$$

where $\omega_{p}=\sqrt{4 \pi e^{2} n_{e} / m_{e}}$ is the plasma frequency and $\nu$ is the electron-ion collision frequency. The heating rate per unit volume in the cluster becomes

$\frac{\partial U}{\partial t}=\frac{9 \omega^{2} \omega_{p}^{2} \nu}{8 \pi} \frac{1}{9 \omega^{2}\left(\omega^{2}+\nu^{2}\right)+\omega_{p}^{2}\left(\omega_{p}^{2}-6 \omega^{2}\right)}\left|E_{0}\right|^{2}$.

Note that when the electron density inside the cluster sphere is high, i.e., when $n_{e} / n_{\text {crit }} \gg 3$ (where the critical electron density is $n_{\text {crit }}=m_{e} \omega^{2} / 4 \pi e^{2}$ ), then the field inside the cluster, given by Eq. (16), is smaller than the field surrounding the cluster in vacuum. This shielding results in a decrease of the heating rate since the laser electric field energy density is shielded from the cluster interior. However, when $n_{e} / n_{\text {crit }}=3,|\varepsilon+2|$ goes through a minimum. At this point the field and the heating rate inside the cluster are enhanced with respect to the surrounding field. The relative height and width of this resonance are determined by the value of the collision frequency $\nu$. The form of the heating rate from Eq. (19) as a function of $n_{e}$ for fixed values of the collision frequency $\nu$ is shown in Fig. 5. As the electron density in the

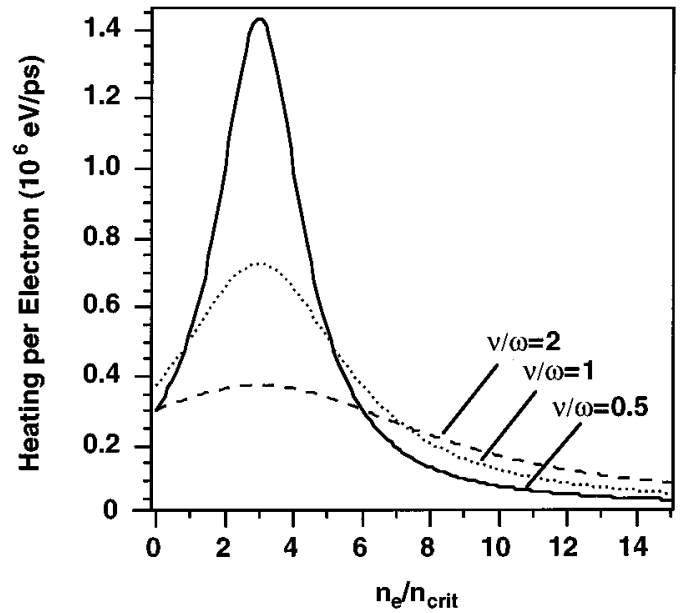

FIG. 5. Calculated heating rate in a cluster by an 825-nm laser pulse with a peak intensity of $10^{16} \mathrm{~W} / \mathrm{cm}^{2}$ as a function of $n_{e}$ for three values of the collision frequency $\nu$, using Eq. (19).

cluster increases, the heating rate peaks and then falls below the vacuum heating rate when $n_{e} / n_{\text {crit }}>\sim 5$.

The electron-ion collision frequency can be described by the standard Coulomb formulas of Silin [35]. These frequencies have analytic formulas for the extreme cases in which the electron oscillation velocity $v_{\text {osc }}$ is much larger than and much smaller than the electron thermal velocity $v_{k T}$. When the oscillation velocity is small the collision frequency is given by

$$
\nu=\frac{4}{9}\left(\frac{2 \pi}{3}\right)^{1 / 2} \frac{Z^{2} e^{4} n_{i}}{m_{e}^{1 / 2}\left(k T_{e}\right)^{3 / 2}} \ln \Lambda, \quad v_{\mathrm{osc}} \ll v_{k T}
$$

while the opposite case is given by

$$
\nu=\frac{16 Z^{2} e n_{i} m_{e} \omega^{3}}{E_{0}^{3}}\left(\ln \left[\frac{e E_{0}}{2 m_{e} \omega \nu_{e}}\right]+1\right) \ln \Lambda, \quad v_{\mathrm{osc}} \gg v_{k T} .
$$

Here, $\ln \Lambda$ is the standard Coulomb logarithm. To find the collision frequency for the intermediate case when $v_{\text {osc }} \approx v_{k T}$ we numerically integrate the general equation describing the collision frequency in Ref. [35].

At high densities, however, the equations of Silin predict collision frequencies that are too large (i.e., $\nu \gg \omega$ ). It is therefore necessary to clamp $\nu$ at some reasonable value. For our calculations we use a harmonic average of the Silin frequency with some suitably chosen maximum collision frequency:

$$
\frac{1}{\nu}=\frac{1}{\nu_{\text {Coul }}}+\frac{1}{\nu_{\max }}
$$

We take $1 / \nu_{\max }$ to be equal to the minimum time it would take for an electron driven by the laser field to traverse the distance between two ions a distance $n_{i}^{-1 / 3}$ apart. This maximum collision frequency is given by

$$
\nu_{\max } \cong \frac{2 e E n_{i}^{1 / 3}}{\pi m_{e} \omega}
$$


where $E$ is the field inside the cluster [given by Eq. (16)]. This maximum collision frequency is typically on the order of the laser frequency $\omega$ for solid density plasma. For example, a plasma with an ion density of $10^{22} \mathrm{~cm}^{-3}$ in a field corresponding to an intensity of $10^{15} \mathrm{~W} / \mathrm{cm}^{2}$ has a maximum collision frequency for an 800-nm laser of $\nu_{\max } \sim 4 \omega$. From Eq. (19) and Fig. 5 it is clear that the collisional heating of the cluster can be quite high. This solid density will only be maintained for a short time, however, since the cluster will begin to expand once it begins to be heated.

\section{Cluster expansion mechanisms}

There are two forces which act on the cluster, causing it to expand during and after the laser pulse. The first is the pressure associated with the hot electrons. The heated electrons expand and pull the cold, heavy ions outward with them. The characteristic speed for this expansion is the plasma sound speed:

$$
v_{\text {expand }} \sim\left(\frac{Z k T_{e}}{m_{i}}\right)^{1 / 2}
$$

The other force acting on the cluster results from a charge buildup on the cluster. The hottest electrons in the cluster will have a mean free path large enough that they can free stream directly out of the cluster, and, if the electron's energy is large enough to overcome the space-charge buildup on the cluster, they will leave the cluster altogether. If the charge buildup is sufficiently large, the cluster will undergo a Coulomb explosion in a manner analogous to photoionization of molecules.

We can calculate the rate of expansion by the cluster by considering the conservation of energy of an expanding fluid sphere that maintains a uniform (but decreasing) density throughout the sphere during the expansion [36]:

$$
P 4 \pi r^{2} \frac{\partial r}{\partial t}=\frac{\partial K_{c}}{\partial t} .
$$

Here $P$ is the total pressure associated with the surface of the sphere and $K_{c}$ is the kinetic energy associated with the expanding cluster particles. If we ignore the pressure of the cold ions, $P$ can be broken down into a hydrodynamic term from the electron pressure and a suitably chosen pressure from the Coulomb force:

$$
P=P_{e}+P_{\text {Coul }} \text {. }
$$

The equation for the radius of the cluster becomes

$$
\frac{\partial^{2} r}{\partial t^{2}}=3 \frac{P_{e}+P_{\text {Coul }}}{n_{i} m_{i}} \frac{1}{r} .
$$

The hydrodynamic portion of the cluster expansion results from a conversion of electron thermal energy to directed kinetic energy. The effects that the expansion has on the electron temperature can be calculated by equating the rate of change of radial kinetic energy from the thermal contribution with the rate of change of thermal energy within the cluster:

$$
P_{e} 4 \pi r^{2} \frac{\partial r}{\partial t}=-\frac{3}{2}\left(\frac{4}{3} \pi r^{3}\right) n_{e} k \frac{\partial T_{e}}{\partial t}+\frac{\partial E_{\mathrm{coll}}}{\partial t}
$$

where $\partial E_{\text {coll }} / \partial t$ is the energy deposition due to the collisional heating. Since the electron pressure is given by

$$
P_{e}=n_{e} k T_{e}
$$

the rate of temperature decrease from the cluster expansion is

$$
\left.\frac{\partial T_{e}}{\partial t}\right|_{\exp }=-2 \frac{T_{e}}{r} \frac{\partial r}{\partial t} .
$$

To estimate the force of expansion due to a charge buildup we shall treat the cluster plasma sphere as a perfect conductor and assume that the accumulated charge $Q e$ on the cluster resides on the surface. The stored energy of the spherical "capacitor" is

$$
E_{Q e}=\frac{Q^{2} e^{2}}{2 r},
$$

which implies that the force per unit area on the surface of the cluster is

$$
P_{\text {Coul }}=\frac{Q^{2} e^{2}}{8 \pi r^{4}}
$$

From the $1 / r^{4}$ scaling in Eq. (32) we see that the Coulomb force becomes most important for small clusters. It is informative to compare the relative contributions of these forces on the cluster for parameters of interest to determine which will be dominant. Comparing Eqs. (29) and (32) we see that for a $100-\AA$ cluster with an electron density of $10^{23} \mathrm{~cm}^{-3}$ and a temperature of $1000 \mathrm{eV}$ the Coulomb pressure will become comparable to the hydrodynamic pressure when $Q \approx 10^{5}$. This corresponds to only $\sim 20 \%$ of the electrons having exited the volume (for $Z=8$ ). This implies that it is possible that the Coulomb force may be an important contributor to the cluster expansion rate. Once the cluster expands, however, the hydrodynamic force will begin to dominate since the hydrodynamic pressure scales as $1 / r^{3}$ (through $n_{e}$ ) while the Coulomb force scales as $1 / r^{4}$.

To calculate accurately the time-dependent Coulomb pressure it is necessary to calculate the rate of electrons leaving the cluster volume. This rate needs to account for the collisionality of the plasma and the shape and size of the cluster. Instead of imposing an arbitrary flux limiter on the free streaming rate, as is customary, we estimate the rate by accounting for the geometry of the cluster and the magnitude of the electron mean free path within the cluster. To account for the collisionality of the cluster plasma we shall assume that to leave the cluster volume, the electron needs to be within less than one mean free path, $\lambda_{e}$, of the surface of the cluster sphere. To account for the space-charge buildup on the cluster we assume an electron can escape the cluster only if its kinetic energy exceeds the Coulomb potential energy at the cluster surface.

The rate of electrons exiting the cluster can then be written as 


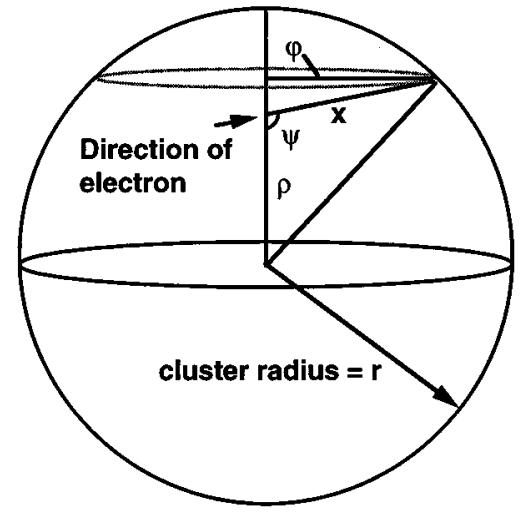

FIG. 6. Geometry of the free streaming rate integral, Eq. (35).

$$
W_{\mathrm{FS}}=\int v f(v) d A d v
$$

where $f(v)$ is the velocity distribution function of the electrons in the cluster which we take to be Maxwellian:

$$
f(v)=4 \pi n_{e}\left(\frac{m_{e}}{2 \pi k T_{e}}\right)^{3 / 2} v^{2} \exp \left[\frac{-m_{e} v^{2}}{2 k T_{e}}\right]
$$

The integral in Eq. (33) over velocity is constrained to include only those velocities that are high enough to escape the Coulomb attraction of the cluster. The integration over the cluster surface is constrained to include only those electrons whose path is within one mean free path of the cluster surface. Accounting for the volume of the cluster encompassed by this constraint, the integral in Eq. (33) can be written

$$
\begin{aligned}
W_{\mathrm{FS}}= & 4 \pi r^{2} \int_{\nu_{\mathrm{esc}}}^{\infty} \int_{\rho_{\min }}^{r} \int_{0}^{2 \pi} \int_{\cos \psi_{\max }}^{\cos \psi_{\min }} v f(v) \frac{3 \rho^{2}}{4 \pi r^{3}} \\
& \times d \cos \psi d \phi d \rho d v .
\end{aligned}
$$

The geometry of this integral is shown in Fig. 6. The free streaming constraints can be written as

$$
\begin{gathered}
\cos \psi_{\max }=-1, \\
\cos \psi_{\min }=\frac{\rho^{2}+\lambda_{e}^{2}-r^{2}}{2 \rho \lambda_{e}}, \\
\rho_{\min }=r-\lambda_{e}, \\
x<\lambda_{e} .
\end{gathered}
$$

Evaluation of Eq. (35) with these constraints yields for the free streaming rate

$$
\begin{aligned}
W_{\mathrm{FS}}= & n_{e} \frac{2 \sqrt{2 \pi}}{m_{e}^{1 / 2}\left(k T_{e}\right)^{1 / 2}}\left(K_{\mathrm{esc}}+k T_{e}\right) \exp \left[-\frac{K_{\mathrm{esc}}}{k T_{e}}\right] \\
& \times\left\{\begin{array}{ll}
\frac{\lambda_{e}}{4 r}\left(12 r^{2}-\lambda_{e}^{2}\right), \quad \lambda_{e}<2 r \\
4 r^{2}, \quad \lambda_{e}>2 r
\end{array}\right\}
\end{aligned}
$$

where $K_{\text {esc }}$ is the minimum kinetic energy required for the electron to overcome any charge buildup on the cluster. Note that when $\lambda_{e}>2 r$ and $K_{\mathrm{esc}}=0$ this formula reduces to the normal free streaming rate of electrons from a uniform sphere. For the electron mean free path in the cluster plasma we use the standard Spitzer formula [37]:

$$
\lambda_{e}=\frac{\left(k T_{e}\right)^{2}}{4 \pi n_{e}(Z+1) e^{4} \ln \Lambda} .
$$

To estimate the electron escape energy we assume that the cluster sphere is a perfect conductor and that any built-up charge, therefore, resides on the surface of the sphere. Consequently, the electron escape kinetic energy is set equal to the Coulomb potential energy of the electron at the cluster sphere surface,

$$
K_{\mathrm{esc}}=\frac{(Q+1) e^{2}}{r} .
$$

This model assumes that the electron energy distribution function is Maxwellian at all times. This is not strictly correct since the majority of the free streaming electrons will come from the hot tail of the Maxwellian. It will take a finite time for the energy distribution to thermalize and repopulate the distribution with the most energetic electrons. Consequently, our treatment will overestimate the rate of free streaming. However, we find that the charge buildup on the cluster is not important in determining the expansion dynamics of the large clusters considered in this study. Only when we consider small clusters $(<50 \AA)$ does the free streaming become important.

Whether the expansion is through hydrodynamic forces or Coulomb forces, the expansion of the cluster will be quite rapid once significant heating has taken place. It is clear from this analysis, however, that large clusters will expand more slowly than small clusters. Thus larger clusters, in general, are superior for absorption since they will hold together longer during heating by the laser pulse.

The expansion has an effect on the cluster dynamics by cooling the electron temperature through Eq. (30). We need to consider another mechanism for cooling the electrons as well. This cooling comes from an exchange of thermal energy from the hot electrons to the cold ions through collisional thermalization.

\section{Electron-ion thermalization in the cluster}

Coulomb collisions of energetic electrons can transfer thermal energy to the cold ions in the cluster plasma. This process can be described by a thermal equilibration rate which can be written as [37]

$$
\left.\frac{\partial T_{e}}{\partial t}\right|_{\mathrm{eq}}=-\frac{T_{e}-T_{i}}{\tau_{\mathrm{eq}}} .
$$

The electron-ion equilibration time $\tau_{\mathrm{eq}}$ is given by [37]

$$
\tau_{\mathrm{eq}}=\frac{3 m_{e} m_{i}}{8 \sqrt{2 \pi} n_{i} Z^{2} e^{4} \ln \Lambda}\left(\frac{k T_{e}}{m_{e}}+\frac{k T_{i}}{m_{i}}\right)^{3 / 2} .
$$


From this formula, we see, for example, that for a cluster with $Z=8$ and an electron temperature of $1 \mathrm{keV}$ (with cold ions) this equilibration time is approximately $30 \mathrm{ps}$. Therefore this is a relatively unimportant mechanism on the time scale of the cluster heating by the laser pulse, which occurs on a $\sim 1$-ps time scale. The predominant mechanism for coupling energy from the electrons to the ions is through the radial expansion.

\section{NUMERICAL MODELING OF THE LASER-CLUSTER INTERACTION}

To explore the interplay of the various processes described in the preceding section we have developed a numerical code that solves for the cluster parameters subject to these processes. Our code self-consistently solves the time dependence of the ionization stages of the cluster atoms and the respective electron and ion densities (assumed to be uniform throughout the cluster) by numerically integrating the ionization rates of Eqs. (6), (7), and (13). These processes are calculated by assuming that the electric field inside the cluster is shielded according to Eq. (16). The cluster radius is found using Eq. (27). The heat deposition of the laser into the cluster is found using Eq. (19) with appropriately chosen collision frequencies as a function of the cluster plasma conditions. The electron temperature is calculated using this heat deposition rate, the electron cooling associated with the cluster expansion, given by Eq. (30), and the transfer of energy to the ions, given by Eq. (40). Finally, the time dependence of the charge buildup on the cluster is found from Eq. (37) and the pressure used in Eq. (27) is adjusted accordingly.

Since the experimental data are largely dependent on the electron temperature of the underdense plasma that results after the clusters have expanded, we calculate the electron temperature by including not only the random thermal energy the electrons retain with respect to the spherical cluster after expansion but also include the average of the radial expansion velocity. We assume that after expansion, the electrons interact with the electrons from other clusters and the directed radial velocity of the electrons is reconverted into random thermal energy through electron-electron collisions in the underdense bulk plasma. Thus we calculate the cluster parameters until the density of the ions in the cluster has dropped because of the cluster expansion to a value that is characteristic of the bulk underdense plasma. Since we have assumed a uniform expansion by the cluster, the electron radial velocity linearly increases from zero as the radial position increases in the cluster. The electron energy is therefore found by averaging over the cluster sphere and is given by

$$
\langle K\rangle=\frac{3}{2} k T_{e}+\frac{3}{10} m_{e} v_{\text {expand }}^{2},
$$

where $v_{\text {expand }}$ is the radial velocity of the cluster boundary. The bulk plasma electron temperature is then found from the relation $k T_{e}=\frac{2}{3}\langle K\rangle$.

An example of calculated cluster parameters is shown in Fig. 7. This calculation is for a $100-\AA$-diam Ar cluster (initial density equals $1.8 \times 10^{22} \mathrm{~cm}^{-3}$ ) irradiated by a 130 -fs pulse [full width at half maximum (FWHM)] with a wavelength of $825 \mathrm{~nm}$ and peak intensities of $0.5,1.0$, and $2.0 \times 10^{16}$ $\mathrm{W} / \mathrm{cm}^{2}$. Figure 7(a) shows the laser pulse profile. Figure 7(b)

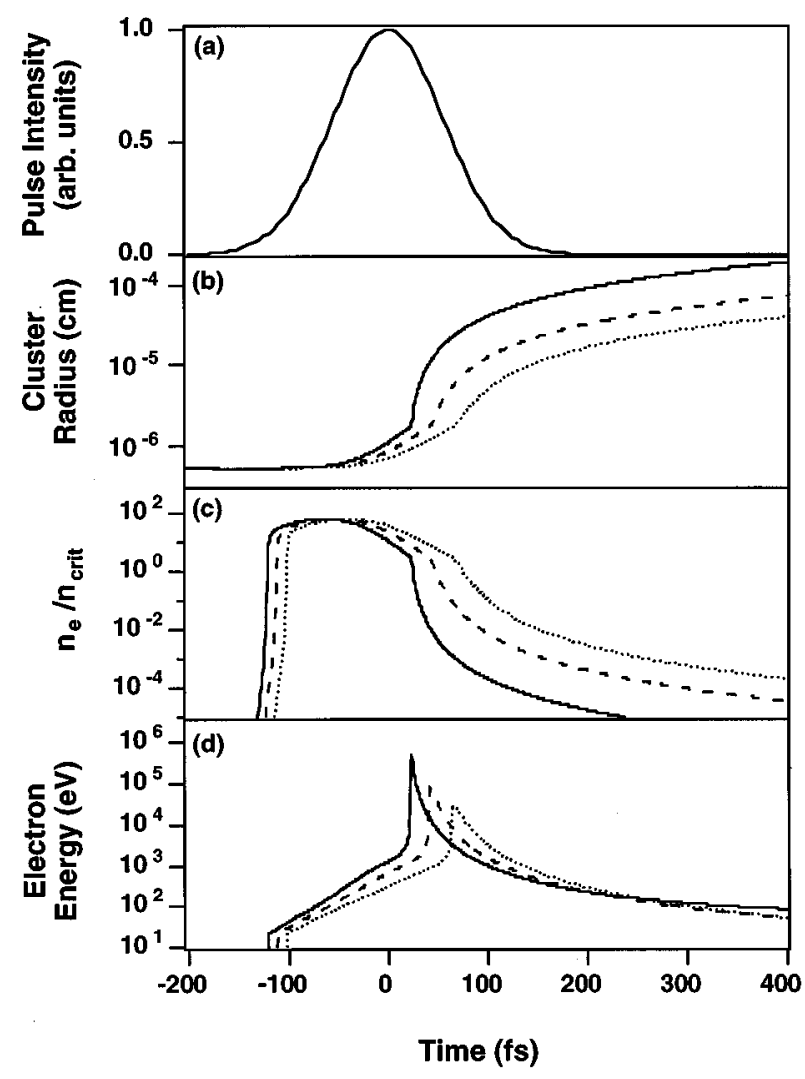

FIG. 7. Simulation results for a $100-\AA$ Ar cluster subject to a 130 -fs, $825-\mathrm{nm}$ laser pulse $\left(n_{\text {crit }}=1.6 \times 10^{21} \mathrm{~cm}^{-3}\right)$ with three different peak intensities, $0.5 \times 10^{16} \mathrm{~W} / \mathrm{cm}^{2}$ (dotted line), $1.0 \times 10^{16}$ $\mathrm{W} / \mathrm{cm}^{2}$ (dashed line), and $2.0 \times 10^{16} \mathrm{~W} / \mathrm{cm}^{2}$ (solid line). (a) Pulse intensity profile. (b) Cluster radius. (c) Cluster electron density. (d) Electron energy.

shows the radius of the cluster as a function of time, Fig. 7(c) shows the electron density, and Fig. 7(d) shows the average electron energy [defined by Eq. (42)] as a function of time.

Initially the electron density rises rapidly from zero during the leading edge of the laser pulse due to the onset of tunnel ionization. This occurs early in the laser pulse when the intensity is approximately $2 \times 10^{14} \mathrm{~W} / \mathrm{cm}^{2}$, the point at which tunnel ionization becomes significant for Ar. This rate is then quenched since the electric field is shielded by the formation of a high-density plasma in the cluster. The plasma begins to slowly heat and expand. After the plasma shielding becomes significant, the dominant ionization mechanism becomes the electron collisional ionization. The electron temperature rises to a few hundred $\mathrm{eV}$, accelerating the cluster expansion rate. When the electron density has dropped due to expansion to a value such that $n_{e} / n_{\text {crit }}=3$, the field is enhanced, the heating rate rises quickly, and the electron energy jumps. This gives rise to the spikes seen in the electron temperature in Fig. 7(d). This rapid increase in temperature is followed by an accelerated rate of expansion and the electron energy drops as the electrons give some of their energy to the much more massive ions in the expansion process. After the pulse has passed the electrons retain about $500-2000 \mathrm{eV}$ of energy.

This calculation illustrates the importance of the cluster expansion and the electric field enhancement in determining 


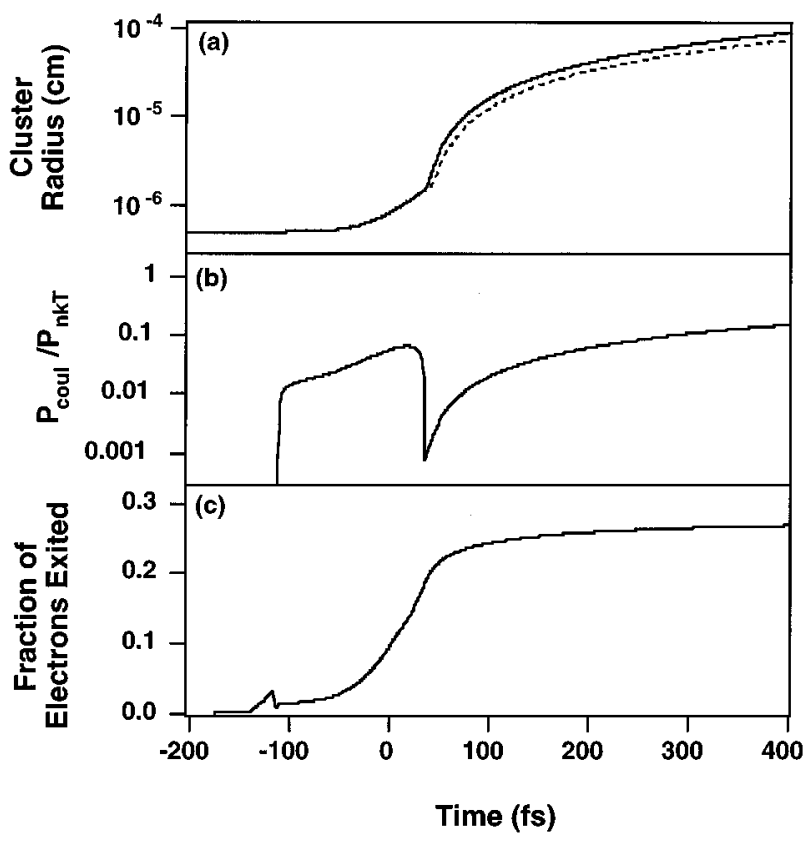

FIG. 8. Simulation results for a $100-\AA$ Ar cluster subject to a 130 -fs, $825-\mathrm{nm}$ laser pulse with a peak intensity, $1.0 \times 10^{16} \mathrm{~W} / \mathrm{cm}^{2}$. (a) Cluster radius without a Coulomb expansion force (dashed line) and with the Coulomb expansion force (solid line). (b) Ratio of the Coulomb expansion pressure to the hydrodynamic expansion pressure. (c) Fraction of cluster electrons exited the cluster sphere by free streaming.

the cluster electron heating. The phenomenon of increased laser energy absorption when $n_{e} / n_{\text {crit }}=3$ is essentially equivalent to the well-known fact that when a laser illuminates a solid the laser absorption occurs predominantly at the point in the electron density profile where $n_{e} \sim n_{\text {crit }}$ [7]. With an electron density higher than $n_{\text {crit }}$ the field is shielded, lowering the net heating rate. At lower density, the collisional frequency is lower and, consequently, the heating rate drops. This is also true of the cluster, however, the exact location of the peak in the heating rate has shifted to $3 n_{\text {crit }}$ due to the geometry of the plasma. From this analysis we see that the cluster expansion is actually quite important in determining the electron temperatures attainable since it is the passage of the cluster density through the region of $n_{e} \sim 3 n_{\text {crit }}$ that produces the majority of laser energy deposition.

We should also point out that under the conditions considered in Fig. 7, the Coulomb expansion force does not play a significant role in the cluster dynamics. Figure 8(a) compares the calculated radius of the cluster as a function of time for the case when Coulomb expansion effects are included with the case when they are neglected (laser intensity equals $10^{16} \mathrm{~W} / \mathrm{cm}^{2}$ ). There is only a slight difference (less than a factor of 2) between the cluster radii of the two cases. Figure 8(b) shows the ratio of the Coulomb pressure [Eq. (32)] to that of the electron thermal pressure [Eq. (29)] as a function of time. This quantity never exceeds 0.1 , indicating that it is the hydrodynamic pressure which is most important in determining the cluster expansion rate. The calculated fraction of electrons that have left the cluster by free streaming is plotted in Fig. 8(c). In the early stages of the cluster heating this fraction remains below $10 \%$. Only after the rapid heating

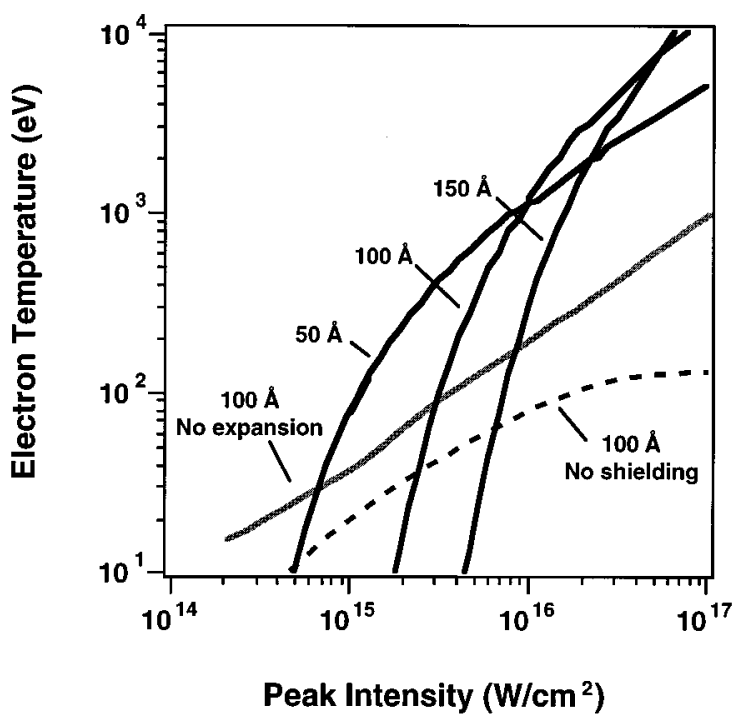

FIG. 9. Calculated electron temperature of electrons in Ar clusters heated by a 130 -fs 825 -nm laser after the clusters have expanded as a function of laser peak intensity.

resulting from the passage of the cluster resonance does the free streaming rate become significant, with the fraction of electrons exited rising to $25 \%$. By this time, however, the cluster has expanded significantly and the Coulomb expansion term remains unimportant.

The situation is somewhat different for smaller clusters. When the cluster is smaller $(<50 \AA)$ the free streaming rate is more important (due to the higher surface to volume ratio) and the contribution to the expansion by the Coulomb expansion is the dominant term. In a sense, this means that small clusters expand like large molecules in an intense laser field, via Coulomb explosion, while large clusters behave in a manner characteristic of a solid density plasma.

These calculations indicate that electron temperatures $>1000 \mathrm{eV}$ are achievable when large clusters are illuminated by pulses with peak intensity $>10^{16} \mathrm{~W} / \mathrm{cm}^{2}$. Figure 9 shows the calculated electron temperature [once again defined as $\left.k T_{e}=\frac{2}{3}\langle K\rangle\right]$ as a function of peak laser intensity for a 130-fs, 825-nm pulse illuminating an Ar cluster. (The calculated temperatures of clusters of other species are comparable.) The calculated temperature for three different cluster sizes is shown, 50, 100, and $150 \AA$. In all cases the electron temperature exceeds $1 \mathrm{keV}$ when the laser pulse intensity is $>10^{16}$ $\mathrm{W} / \mathrm{cm}^{2}$. For comparison, the electron temperature has been calculated for the case when a $100-\AA$ Ar cluster is heated but not allowed to expand. Also shown in this figure is the calculation for the case in which the cluster is allowed to expand normally but the field inside the cluster is set equal to the field outside the cluster at all times during the laser pulse (i.e., no shielding). At the high intensities, these calculated temperatures are significantly lower than for the full calculation. This is because, as we showed in Fig. 7, the passage of the cluster through the field resonance enhances the electron temperature. This heating rate enhancement is absent when expansion or shielding is ignored.

At the low intensities, however, the temperature of the cluster is quite low compared to the case in which expansion is ignored. This is because with low peak intensity the initial 


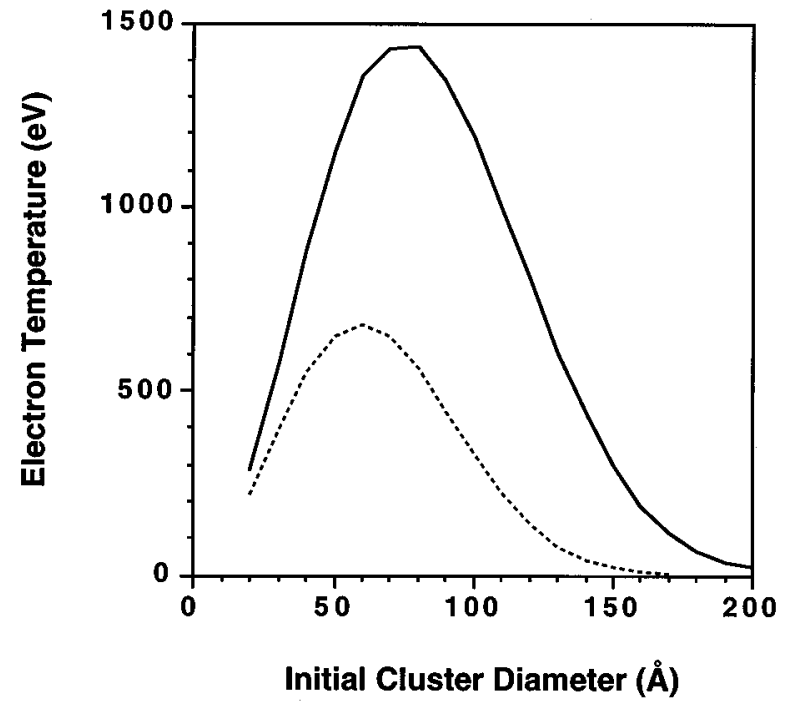

FIG. 10. Calculated electron temperature of electrons in Ar clusters heated by a 130 -fs $825-\mathrm{nm}$ laser with a peak intensity of $5.0 \times 10^{15} \mathrm{~W} / \mathrm{cm}^{2}$ (dashed line), and $1.0 \times 10^{16} \mathrm{~W} / \mathrm{cm}^{2}$ (solid line) after the clusters have expanded, as a function of the initial cluster size.

ionization and heating of the clusters is late in the pulse and the cluster does not expand through the field resonance until after the pulse has passed. This trend can be seen in Fig. 6(d) where the spike in the electron temperature comes later and is smaller as the intensity is decreased. Note that the smaller cluster size $(50 \AA)$ actually exhibits higher temperatures at lower intensity than do the larger clusters. This is merely due to the more rapid expansion rate of the smaller cluster which brings the cluster plasma into resonance during the laser pulse at a lower intensity.

This analysis suggests that an optimum cluster size might exist for optimum heating. The calculated electron temperature after expansion of Ar clusters as a function of initial cluster diameter is shown in Fig. 10 for two intensities, $5 \times 10^{15}$ and $1 \times 10^{16} \mathrm{~W} / \mathrm{cm}^{2}$. The electron temperature exhibits a broad maximum and peaks at around $70 \AA$ for $1 \times 10^{16}$ $\mathrm{W} / \mathrm{cm}^{2}$. Small clusters remain relatively cold due to their rapid expansion which moves the cluster plasma through the heating rate resonance rapidly. Large clusters, on the other hand, do not expand quickly enough to come into resonance during the 130-fs laser pulse.

The high electron temperatures that are achieved in the cluster during the laser pulse also give rise to rapid electron collisional ionization. Consequently, high charge states can be created in the cluster before it disassembles. Figure 11(a) shows the fractional populations of $\mathrm{Ar}$ ions from $\mathrm{Ar}^{5+}$ to $\mathrm{Ar}^{10+}$ as a function of time in a $100-\AA$ Ar cluster irradiated by a pulse with an intensity of $1 \times 10^{16} \mathrm{~W} / \mathrm{cm}^{2}$. Under these conditions it is the thermal ionization that dominates. The threshold for significant tunnel ionization for these charge species (defined as the peak intensity at which $\int W_{\text {tun }} d t=1$ ) is $6 \times 10^{15}, 9 \times 10^{15}, 2 \times 10^{16}, 3 \times 10^{16}, 1 \times 10^{18}, 2 \times 10^{18}$, and $3 \times 10^{18} \mathrm{~W} / \mathrm{cm}^{2}$, respectively. As can be seen in Fig. 11, the dominant charge species created in the cluster plasma are $\mathrm{Ar}^{8+}, \mathrm{Ar}^{9+}$, and $\mathrm{Ar}^{10+}$. A peak intensity 100 times higher than used in the simulation would be required to access these (a)

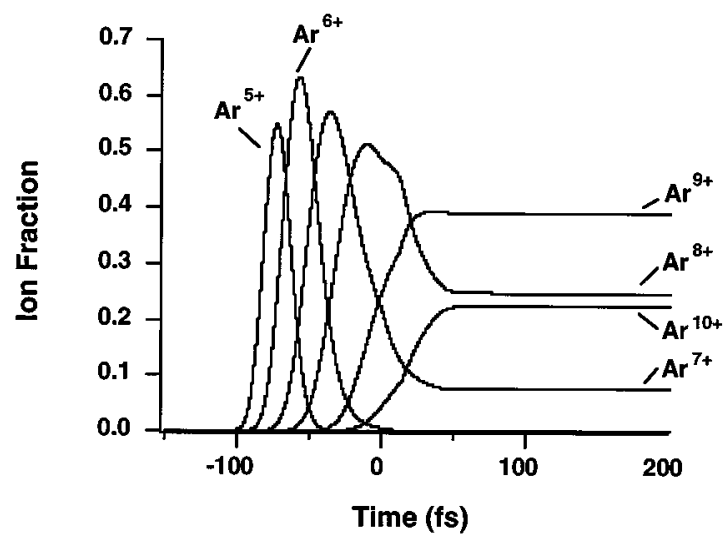

(b)

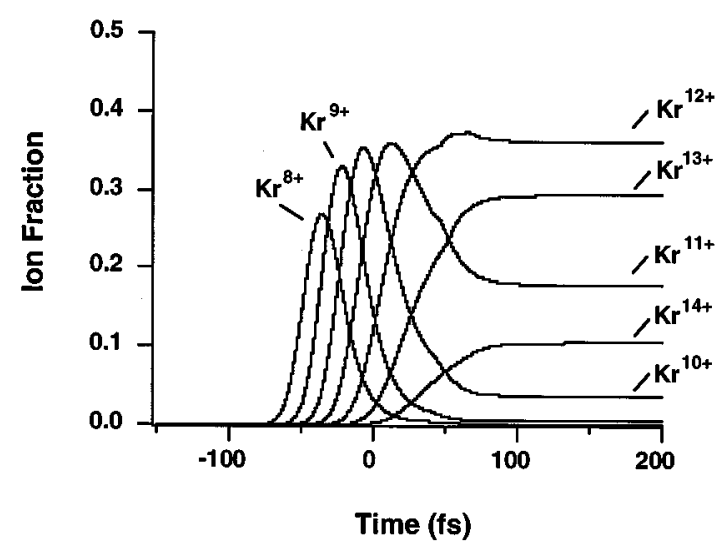

FIG. 11. Calculated fractional populations of (a) Ar ions from $\mathrm{Ar}^{5+}$ to $\mathrm{Ar}^{11+}$ and (b) $\mathrm{Kr}$ ions from $\mathrm{Kr}^{8+}$ to $\mathrm{Kr}^{14+}$ as a function of time in a $100-\AA$ cluster irradiated by a pulse with an intensity of $1.0 \times 10^{16} \mathrm{~W} / \mathrm{cm}^{2}$.

charge states by tunnel ionization. Due to the rapid drop in density as the cluster expands the ionization rate drops and these charge states are effectively "frozen" in the underdense plasma that results after the clusters expand. Calculations of krypton exhibit similar behavior with somewhat higher charge states accessed under similar conditions. Figure 11(b) shows the ionization fraction time histories for $\mathrm{Kr}$ $8-14+$ for a $100-\AA \mathrm{Kr}$ cluster illuminated by an intensity of $1 \times 10^{16} \mathrm{~W} / \mathrm{cm}^{2}$. Here the dominant charge states created are $\mathrm{Kr}^{11+}, \mathrm{Kr}^{12+}, \mathrm{Kr}^{13+}$, and $\mathrm{Kr}^{14+}$. To tunnel ionize to these charge states requires $3 \times 10^{17}, 5 \times 10^{17}, 6 \times 10^{17}$, and $8 \times 10^{17}$ $\mathrm{W} / \mathrm{cm}^{2}$, respectively.

In light of these computational results it is interesting to consider the heating of clusters when compared to heating of solids. The primary difference between the heating of the clusters considered in this work and the heating of a solid target by an intense laser pulse is that the heating of a solid is accompanied by conductive cooling of the hot plasma into the cold, bulk substrate by conductive and free streaming cooling [38]. Heating of the cluster is not hindered by this cooling and might, therefore, be expected to reach temperatures in excess of that for solid targets under similar conditions. As mentioned, the heating in a solid is predominantly at the critical surface. The expansion of the small clusters serves to bring the electron density into a region around the critical density for all the electrons in the plasma for, at least, 
a brief period of time. Consequently, the absorption of the laser light by the clusters should be comparable if not superior to that of a solid for these reasons. The experimental investigation described below seems to confirm this assertion.

The experimental investigations, however, typically observe $\mathrm{x}$-ray emission from the plasma on a time scale longer than we have considered in these calculations $(\geqslant 10 \mathrm{ps})$. Consequently, we also need to consider the dynamics of the underdense plasma to derive information about the temperature and charge state production in the clusters from the experimental data.

\section{PROPERTIES OF AN UNDERDENSE PLASMA HEATED BY LASER IRRADIATION OF CLUSTERS}

After the clusters expand, the properties of the plasma are quite different. The plasma that is created by the passage of the laser pulse through the gas jet will cool and decay on a time scale that is much longer than the expansion time scale of the clusters. If the laser confocal parameter is longer than the width of the gas jet flow, the resulting plasma is roughly cylindrical with a radial dimension that is roughly that of the laser focus (typically $\sim 25-50 \mu \mathrm{m}$ ). To compare the calculations to experiments and to evaluate the cluster plasma as a potential source of soft $x$ rays, it is necessary to evaluate the mechanisms for x-ray production and cooling of the hot, low-density plasma.

The characteristics of the gaseous plasma that results after the irradiation of the clusters include a high state of ionization $(Z>8$ for $\mathrm{Ar}, Z>10$ for $\mathrm{Kr}$ ) and high electron temperatures $(100-1000 \mathrm{eV})$. The plasma emission will be dominated by line emission from the resonance lines of the ions in the plasma. The excited states of these ions can be populated in two ways. First, collisional excitation of the hot electrons can excite the transitions in these ions provided that the electron temperature is sufficiently high for this to be important. Second, recombination of free electrons into the upper states of the ions will be followed by a collisional-radiative cascade into the upper levels of strong radiative transitions [39]. This will be important after the plasma has cooled from its initial hot state by expansion and conductive cooling to the cold gas that surrounds the plasma heated by the laser.

The collisional excitation of the resonance transitions in most ions can be estimated from the simple formula [40]

$$
W_{n n^{\prime}}=\frac{1.6 \times 10^{-5} f_{n n^{\prime}}\langle\vec{g}\rangle}{\Delta E \sqrt{k T_{e}}} \exp \left[-\Delta E / k T_{e}\right] \quad\left(\mathrm{cm}^{3} \mathrm{~s}^{-1}\right)
$$

where $f_{n n^{\prime}}$ is the transition oscillator strength, $\langle\vec{g}\rangle$ is the Gaunt factor $(\approx 0.2$ for ions [40]), and $\Delta E$ is the transition energy (in $\mathrm{eV}$ ). From this relation we see that this excitation rate is significant only when $k T_{e} \sim \Delta E$. For the transitions of energies that are comparable to the initial temperature, this excitation mechanism will only be significant in the early stages of the plasma decay before the plasma has cooled significantly. After the plasma has cooled, three-body recombination becomes important. The rate of recombination can be estimated from the simple formula [41]

$$
W_{\mathrm{recom}}=n_{e} \frac{4 \pi \sqrt{2 \pi}}{9} \frac{Z^{3} e^{10}}{m_{e}^{1 / 2}\left(k T_{e}\right)^{9 / 2}} \ln \Lambda
$$

This rate strongly favors colder plasma because of its strong $\left(k T_{e}\right)^{-9 / 2}$ scaling.

To calculate the time scale for the $\mathrm{x}$-ray emission from a plasma that has been heated by the presence of clusters we have developed a code that calculates the cooling and expansion of the cylindrical plasma. Our calculation includes the effects of both adiabatic expansion by the plasma as well as the conductive cooling of the hot plasma at the center of the laser focus to the colder plasma surrounding the center. The hydrodynamics of the plasma are determined by solving the ion fluid equations subject to electron fluid pressure gradients:

$$
\begin{gathered}
\frac{\partial n_{i}}{\partial t}=-u \frac{\partial n_{i}}{\partial r}-\frac{n_{i} u}{r}-n_{i} \frac{\partial u}{\partial r}, \\
\frac{\partial u}{\partial t}=-u \frac{\partial u}{\partial r}-\frac{Z k T_{e}}{n_{e} m_{i}} \frac{\partial n_{e}}{\partial r}-\frac{Z}{m_{i}} k \frac{\partial T_{e}}{\partial r},
\end{gathered}
$$

where $u$ is the radial ion fluid velocity.

The cooling of the electrons in the plasma is determined by the adiabatic expansion as well as the magnitude of the heat flow from conduction, $q$. The electron temperature, which is a function of both $r$ and $t$, in the plasma cylinder is found from the relation

$$
\frac{\partial T_{e}}{\partial t}=-\frac{4 T_{e}}{3 r} u+\frac{2}{3 n_{e}}\left(\frac{q}{r}+\frac{\partial q}{\partial r}\right) .
$$

The heat flow $q$ is found by using the nonlocal heat flow formula of Luciani, Mora, and Virmont [42]. A nonlocal treatment of the heat flow is required since the classical Spitzer heat flow [43] can exceed the physical heat flux limit of free streaming, $q_{\mathrm{FS}}=3 n_{e} k T_{e} v_{k T} / 2$ when the temperature gradients become large. We use the same simplification to the formula of Luciani, Mora, and Virmont for cylindrical coordinates as used by Djaoui and Offenberger [44]. The heat flow $q$ is given by the formula

$$
\begin{aligned}
q(r)= & \int \frac{1}{64 \lambda\left(r^{\prime}\right)} q_{\text {Spitz }}\left(r^{\prime}\right) \exp \left[-\frac{1}{32 n_{e}\left(r^{\prime}\right) \lambda\left(r^{\prime}\right)}\right. \\
& \left.\times \int_{-\infty}^{\infty} n_{e}\left(r^{\prime \prime}\right) d r^{\prime \prime}\right] \max \left(l, r^{\prime} / r\right) d r^{\prime} .
\end{aligned}
$$

Here $q_{\text {Spitz }}=-\kappa \nabla\left(k T_{e}\right)$ is the classical Spitzer heat flow [43]. ( $\kappa$ is the classical thermal conductivity for a plasma.) Djaoui and Offenberger found that the use of this formula in the calculation of underdense plasma temperatures heated by a 12-ps $\mathrm{KrF}$ laser was in good agreement with experimental measurements of the temperature.

In general, we find that the conductive cooling of the plasmas produced by the irradiation of clusters is important in the early stages of the cooling since high temperatures $(\sim 1000 \mathrm{eV})$ can be generated at the laser pulse peak. After this initial cooling by heat conduction, however, the time scale for cooling is dominated by the hydrodynamic expansion of the plasma. To illustrate this we show the tempera- 
(a)

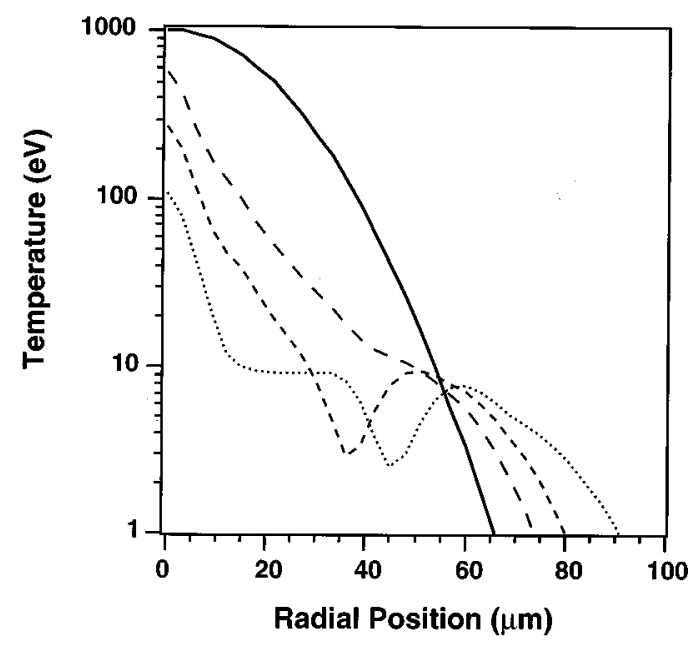

(b)

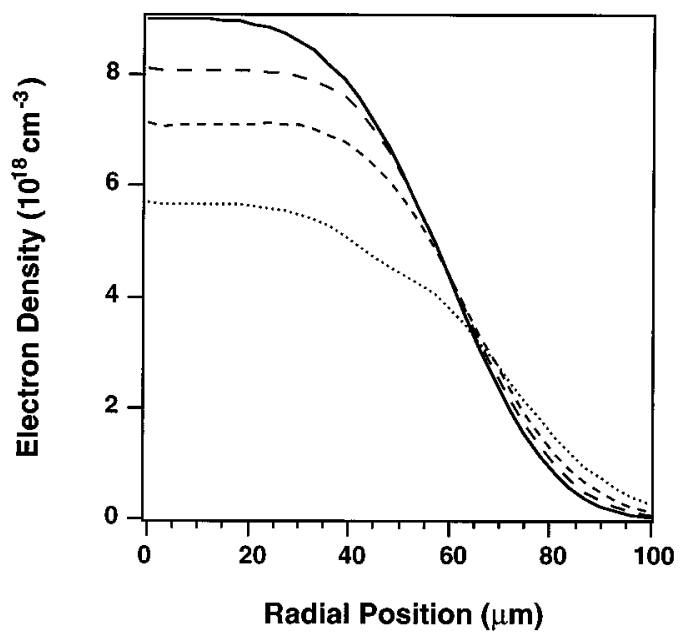

FIG. 12. Calculated temperature (a) and electron density (b) radial profiles for an underdense $\mathrm{Ar}$ gas of initial ion density $1 \times 10^{18} \mathrm{~cm}^{-3}$ that has been heated by the irradiation clusters. The times are $t=0$ (solid line), $500 \mathrm{ps}$ (long dashed line), $1 \mathrm{~ns}$ (short dashed line), and 2 ns (dotted line).

ture and electron density radial profiles for an $\mathrm{Ar}$ gas that has been heated by the irradiation of clusters in Fig. 12. The initial average ion density is taken to be $1 \times 10^{18} \mathrm{~cm}^{-3}$. The initial temperature and electron density profile are found by assuming a focused spot of $100 \mu \mathrm{m}$ in diameter $\left(1 / e^{2}\right)$ and using the calculation of the electron temperature and ionization state as a function of laser intensity for $100-\AA$ Ar clusters detailed in the preceding section. A peak temperature of $1 \mathrm{keV}$ is assumed (corresponding to a peak intensity of about $10^{16} \mathrm{~W} / \mathrm{cm}^{2}$ ). As can be seen from Fig. 12, the center of the temperature distribution drops rapidly between the passage of the laser pulse $(t=0)$ and $500 \mathrm{ps}$. After this time, the temperature cools from the expansion of the plasma on a longer time scale $(>1 \mathrm{~ns})$. This calculation suggests that "warm" plasmas $(>100 \mathrm{eV})$ persist in the plasma for many ns $(>1-2 \mathrm{~ns})$. This is due primarily to the slow rate of cooling associated with the low-density plasma. This is in direct contrast to the time scales of short-pulse-heated solid target plasmas which typically cool on a $<10$-ps time scale by conduction. We therefore expect that a plasma heated by irradiation of clusters will emit $\mathrm{x}$ rays for many ns after the laser has passed.
To determine the actual histories of the ion charge states, it is necessary to solve for the kinetics of the plasma. We do this by using the plasma kinetics code FLY of Lee [45]. This code solves for the time-dependent populations of levels and charge states in the plasma by solving rate equations. The time history of the temperature and density from the hydrodynamics of the plasma is utilized in this code to estimate the actual yields and time history of the $\mathrm{x}$-ray emission from a plasma heated by illumination of the clusters. The results of these calculations will be discussed in the context of the experimental results below.

\section{EXPERIMENTAL APPARATUS}

We have detailed the theory of the interaction of an intense, 100-fs laser with atomic clusters in a gas jet and shown how these interactions can give rise to high charge state production, hot plasmas, and short wavelength generation. To explore these predictions we have conducted a number of experiments. In these experiments, a 130 -fs laser is focused into the plume of a gas jet and the plasma produced is characterized by performing spectroscopy on the resulting $\mathrm{x}$-ray emission.

The laser used for these experiments is a femtosecond, chirped pulse amplification laser utilizing $\mathrm{Cr}_{\mathrm{LiSrAlF}} \mathrm{L}_{6}$ (LiSAF) as the gain medium. This laser has been described in detail previously $[46,47]$. In brief, the front end of the laser uses a self-mode-locked Ti:sapphire oscillator to produce 110 -fs pulses at a wavelength of $825 \mathrm{~nm}$. These pulses are stretched to $450 \mathrm{ps}$ and amplified to the $10-\mathrm{mJ}$ energy level by a LiSAF ring regenerative amplifier. These pulses are further amplified in a 4-mm LiSAF rod, two 9-mm rods, and a $19-\mathrm{mm}$ rod. Up to $1.7 \mathrm{~J}$ of energy has been extracted from this amplifier chain, however, the experiments were typically operated with less than $1 \mathrm{~J}$ from this chain to minimize the nonlinear distortion of the laser pulse. The amplified pulses are then compressed to near-transform-limited 130 -fs pulses with energy up to $0.5 \mathrm{~J}$ for these experiments.

The laser pulses are focused into a vacuum chamber with an $f / 25 \mathrm{MgF}_{2}$ lens $(f=100 \mathrm{~cm})$. The focal spot of this lens contains roughly half of the energy in a near-diffractionlimited $40 \times 50 \mu \mathrm{m}^{2}$ spot. The remainder of the energy surrounds the central spot in a disk of approximately $120 \mu \mathrm{m}$ in diameter. The spot can be roughly approximated for purposes of modeling by a $100-\mu \mathrm{m}$-diam $1 / e^{2}$ spot. The peak intensity is roughly $10^{17} \mathrm{~W} / \mathrm{cm}^{2}$ with $0.5 \mathrm{~J}$ of energy focused into the chamber.

The laser is focused into the output of a supersonic gas jet nozzle. The gas jet used in these experiments is a Mach 8 Laval nozzle that produces atom densities of $0.5-5 \times 10^{18}$ atoms $/ \mathrm{cm}^{3}$ for backing pressures of 100-900 psi [48]. For all experiments the laser was focused $\sim 1 \mathrm{~mm}$ from the nozzle output, which is $1.4 \mathrm{~cm}$ downstream from the jet throat. This jet was chosen because it is predicted to produce large clusters in the heavier noble gases for the backing pressures with which we operate (100-700 psi). We can estimate the expected cluster size by calculating the Hagena parameter, Eq. (1), for our experimental conditions. The jet throat diameter is $150 \mu \mathrm{m}, \alpha$ is $\sim 5^{\circ}$ for our jet nozzle, and $T_{0}$ was room temperature $(\sim 300 \mathrm{~K})$. This parameter varied from $\sim 1 \times 10^{4}$ to $1 \times 10^{5}$ for $\mathrm{Ar}$ and $\sim 2 \times 10^{4}$ to $2 \times 10^{5}$ for $\mathrm{Kr}$ backing our 


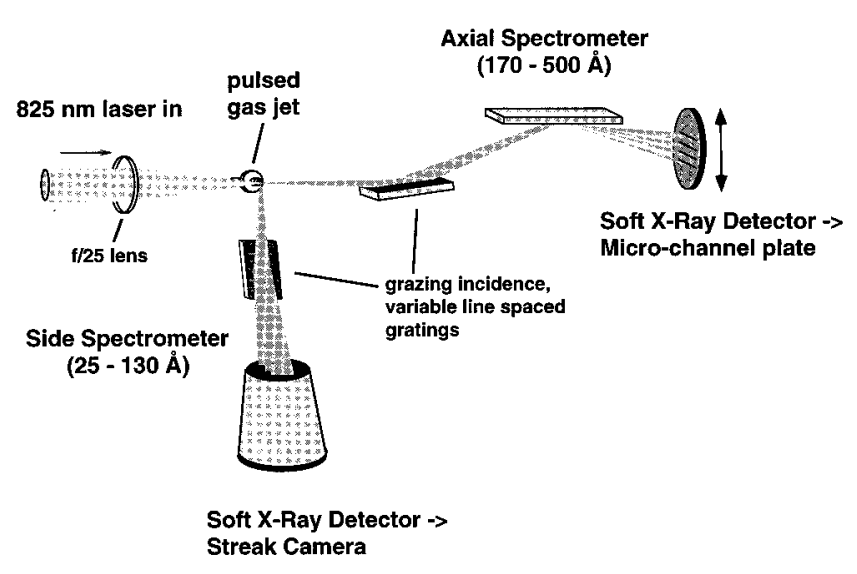

FIG. 13. Schematic of the experimental setup showing the soft$\mathrm{x}$-ray diagnostics.

jet with 100-600 psi, respectively. It never exceeded 5000 for $\mathrm{Ne}$ and $\mathrm{He}$ in our experiments.

A schematic of the experimental apparatus used to diagnose the plasmas is shown in Fig. 13. Through the use of two spectrometers, we were able to monitor the soft-x-ray emission from 500 down to $25 \AA$ on each shot. Both spectrometers utilize variable line spaced gold gratings with a grove density of 1200 lines $/ \mathrm{mm}$. One spectrometer monitored x-ray emission along the laser axis in the 500-170- $\AA$ spectral region. A $2000-\AA \mathrm{Al}$ filter blocked the direct laser light for this spectrometer. The second spectrometer monitored emission from 170 down to $25 \AA$ in a direction perpendicular to the laser propagation. The spectral resolution of both spectrometers was roughly $\Delta \lambda / \lambda \sim 10^{-2}$. Either spectrometer could be mounted with either a CsI-coated, 45-mm-diam microchannel plate detector, which yielded simultaneous information on the spectrum and the angular distribution of the radiation on each shot, or a Kentech x-ray streak camera, which permitted simultaneous measurement of the spectrum and the time history of the $\mathrm{x}$-ray radiation. The temporal resolution of the streak camera was limited to approximately $10 \mathrm{ps}$.

An example of data characteristic of the illumination of the gas jet when it is backed with 300 psi of Ar with a laser intensity of $\sim 10^{16} \mathrm{~W} / \mathrm{cm}^{2}$ is shown in Fig. 14. Figure 14(a) shows the time-integrated emission of the x-rays in the 500$170-\AA$ wavelength range in the axial direction. Very strong line emission is observed from the plasma over the entire spectral region. The high-order harmonics generated by the laser, with their narrow angular divergence, are easily distinguishable from the plasma emission, which emits into all directions. A time-resolved measurement under the same experimental conditions is shown in Fig. 14(b). The 23rd, 25th, and 27th harmonics are clearly visible as fast temporal spikes at the time the laser traverses the gas. The line emission over the entire spectral range, on the other hand, lasts for many ns after the laser produces the plasma.

\section{CLUSTER CONDENSATION ONSET AND SIZE MEASUREMENT BY RAYLEIGH SCATTERING}

To experimentally confirm the presence of large clusters in our gas jet we used a technique of Rayleigh scattering $[49,50]$. The experimental setup for these measurements is
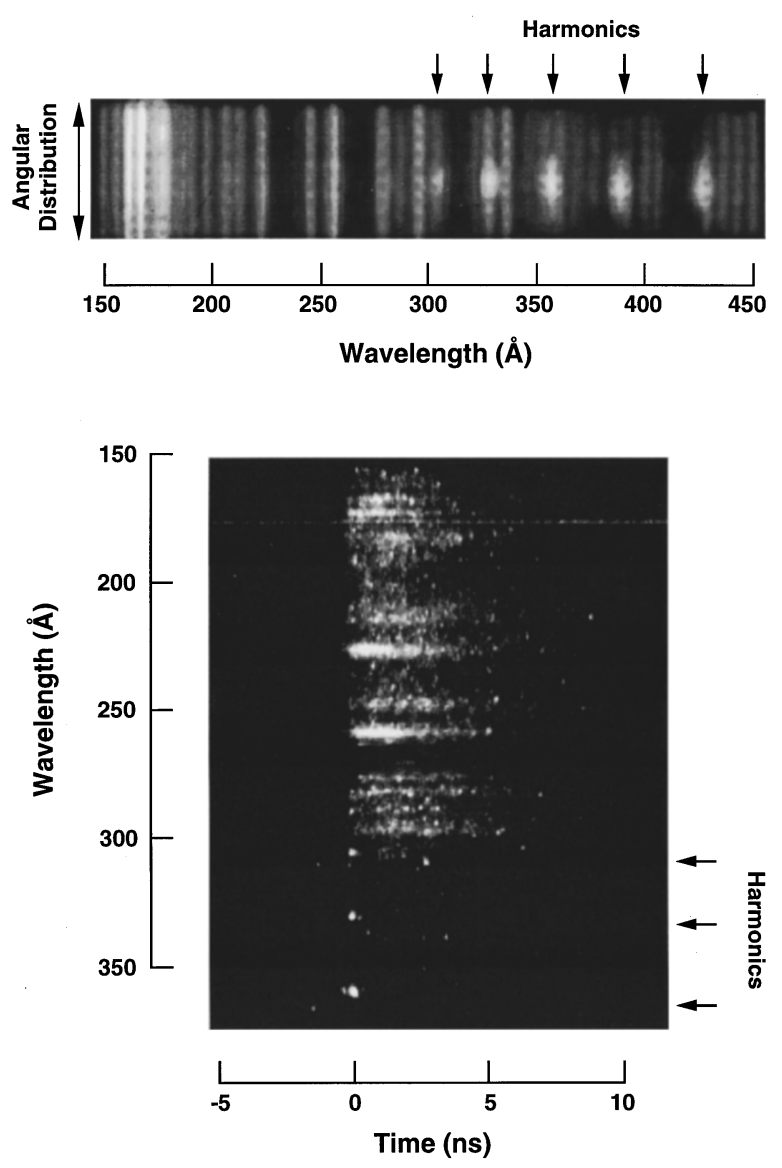

FIG. 14. Soft-x-ray data in the $150-400-\AA$ range characteristic of the illumination of the gas jet when it is backed with 300 psi of Ar with a laser intensity of $\sim 10^{16} \mathrm{~W} / \mathrm{cm}^{2}$. (a) Angularly resolved spectra (the top of the image to the bottom of the image represents $40 \mathrm{mrad}$ divergence). (b) Time-resolved spectra.

shown in Fig. 15. To do this, the gas jet was probed with light from the LiSAF laser that had been frequency doubled in a 3-mm-thick crystal of potassium dihydrogen phosphate (KDP). The second harmonic was used due to the rapid increase in the Rayleigh scattering cross section with decreasing wavelength. The gas at the output of the jet nozzle was irradiated with approximately $1 \mu \mathrm{J}$ of 412 -nm light. The centerline of the approximately 1-mm-diam flow was probed with a beam of approximately $500 \mu \mathrm{m}$ in diameter. The $90^{\circ}$ scattered light was collected with a lens and imaged onto the face of a photomultiplier tube which was covered with an

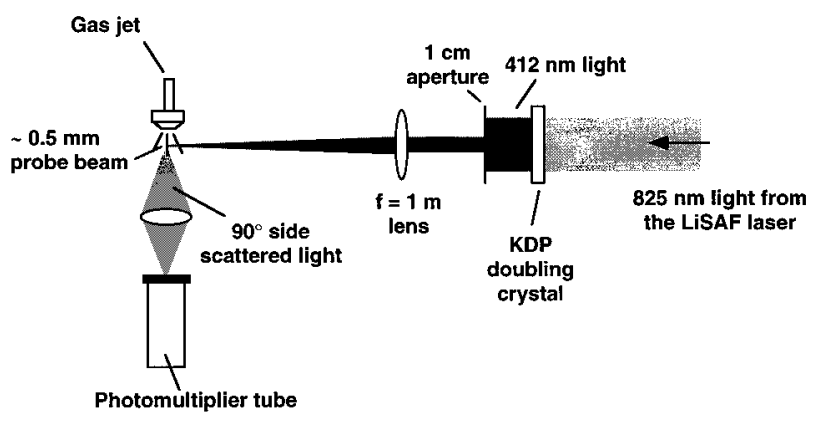

FIG. 15. Experimental setup for Rayleigh scatter measurements of cluster condensation in the gas jet. 
(a)

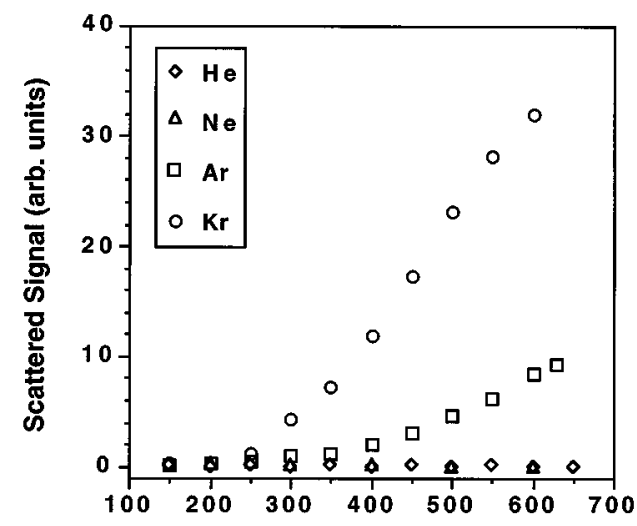

Gas Jet Backing Pressure (psi)

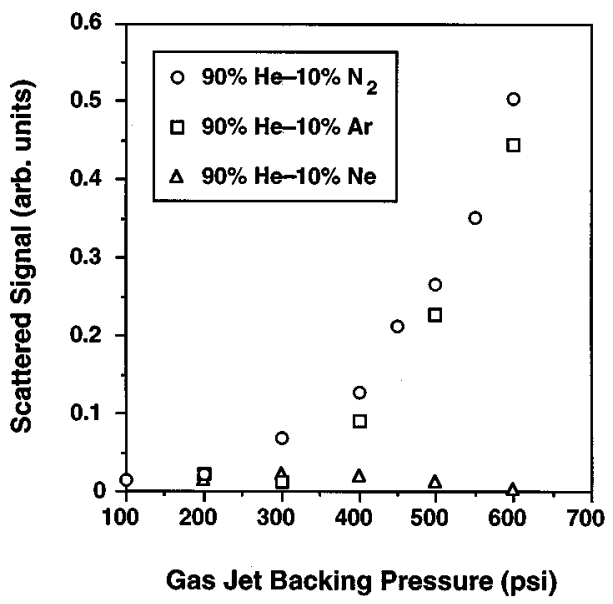

FIG. 16. Measured Rayleigh scattered light signal as a function of backing pressure for (a) pure $\mathrm{He}, \mathrm{Ne}, \mathrm{Ar}$, and $\mathrm{Kr}$, and (b) $10 \%$ mixtures of $\mathrm{Ar}, \mathrm{N}_{2}$, and $\mathrm{Ne}$ with $\mathrm{He}$.

interference filter centered at $412 \mathrm{~nm}$.

Figure 16(a) shows the scattered light signal as a function of backing pressure for $\mathrm{He}, \mathrm{Ne}, \mathrm{Ar}$, and $\mathrm{Kr}$. No significant light scattering above the noise level is observed from either $\mathrm{Ne}$ or He over the range of backing pressures studied. The scattered light signal from the expansion of $\mathrm{Ar}$ and $\mathrm{Kr}$, however, exhibits nonlinear growth with backing pressure, rising above the noise with as little as 150 psi backing the gas jet. This measurement qualitatively confirms our assertion that large clusters are formed in our gas jet when it is backed with $\mathrm{Ar}$ or $\mathrm{Kr}$, and that neither $\mathrm{He}$ nor Ne clusters to any significant degree in the jet.

The nonlinear dependence of the scattered signal is consistent with accepted scaling for the cluster size with backing pressure. Previous measurements of clustering in Ar have shown that the mean number of atoms per cluster, $N_{c}$ scales roughly as $N_{c} \sim p^{2}$ [21]. Since the scattering cross section is given by the classical Rayleigh scattering cross section formula

$$
\frac{d \sigma}{d \Omega}=2 \pi \frac{r^{6}}{\lambda^{4}}\left(\frac{n^{2}-1}{n^{2}+2}\right)
$$

(where $r$ is the radius of the cluster and $n$ is the index of refraction of the cluster material) then we can say that the observed scattered signal should scale as $S \sim n_{\text {clust }} N_{c}^{2}$ where $n_{\text {clust }}$ is the density of clusters in the gas jet. In the regime of large cluster formation (i.e., when $\Gamma^{*}>1000$ ) we can assume that $100 \%$ of the atoms have condensed into clusters [51]. This implies that if $n_{0}$ is the average gas density, which is presumably linear with backing pressure, then $n_{\text {clust }}=n_{0} / N_{c}$. Thus the scattered signal at the highest backing pressures should scale as $S \sim p^{3}$. This is in good agreement with the rise in the Ar data which scales as $S_{\mathrm{Ar}} \sim p^{3.3}$, as well as the $\mathrm{Kr}$ data which grow as $S_{\mathrm{Kr}} \sim p^{3.5}$.

We can make a quantitative estimate for the cluster size based on the scattered light levels. Using Eq. (49) and an extrapolation estimate for the refractive index of the cluster, the signal levels at the highest backing pressure $(600 \mathrm{psi})$ suggest that the mean cluster size is $2-10 \times 10^{4}$ atoms for $\mathrm{Ar}$ and $1-5 \times 10^{5}$ atoms for $\mathrm{Kr}$. Based on the experiment noise level we can put an approximate upper bound on the size of $\mathrm{Ne}$ or He clusters of $<3000$ atoms per cluster, if clusters exist at all in the gas jet. However, estimates for the Hagena parameter for helium suggest that no clusters should form for the backing pressures used in our experiments. The size of the possible error in these estimates of the number of atoms per cluster is large due to a lack of detailed knowledge about the average gas density as well as possible errors in estimating the throughput of our scattered light detection setup. Nonetheless, this corresponds to an accuracy in estimating the cluster diameter of better than $\pm 30 \%$.

For experiments to be described below, we wished to form clusters of the heavier gases while they are mixed with helium. It is well known that the use of $\mathrm{He}$ as a carrier gas will significantly enhance the formation of clusters of heavier atoms in a gas expansion, while the He does not itself undergo clustering [52]. We confirmed the presence of clusters in small fractional mixtures of a heavy gas (fraction $\leqslant 0.1$ ) with a majority of He. Even though the partial pressure for the clustering gases was low, we observe cluster formation in mixtures that contain a heavier gas, as shown in Fig. 16(b). In these data, the $10 \%$ mixtures of $\mathrm{Ar}$ and $\mathrm{N}_{2}$, for example, exhibit nonlinear growth in the scattered Rayleigh signal while a $10 \%$ mixture of $\mathrm{Ne}$ with $\mathrm{He}$ shows no evidence for cluster formation.

\section{SOFT-X-RAY EMISSION CHARACTERISTICS OF THE VARIOUS GASES}

With a confirmation of the presence of large clusters in our experiments we can examine the nature of the x-ray emission from the various gases upon irradiation of the laser. Figure 17 compares the time-integrated soft-x-ray emission from $\mathrm{Ne}, \mathrm{N}_{2}$, Ar, and $\mathrm{Kr}$. In all cases the gas jet was backed with $500 \mathrm{psi}$ of pressure and the incident peak laser intensity was $2 \times 10^{16} \mathrm{~W} / \mathrm{cm}^{2}$. The only signal in Ne comes from very weak harmonics. The signals from the other gases, all gases forming large clusters, exhibit strong line emission across the spectrum. The lines in $\mathrm{N}_{2}$ originate predominantly from $\mathrm{N}^{3+}$, $\mathrm{N}^{4+}, \mathrm{N}^{5+}$, the lines in $\mathrm{Ar}$ originate from $\mathrm{Ar}^{5+}, \mathrm{Ar}^{6+}$, and $\mathrm{Ar}^{7+}$, and the lines in $\mathrm{Kr}$ are largely from $\mathrm{Kr}^{5+}, \mathrm{Kr}^{6+}$, and $\mathrm{Kr}^{7+}$ [53]. In general the time-integrated signal levels from these three gases are roughly equivalent in this range.

The time histories of select lines from these gases under identical conditions are compared in Fig. 18. Here we com- 


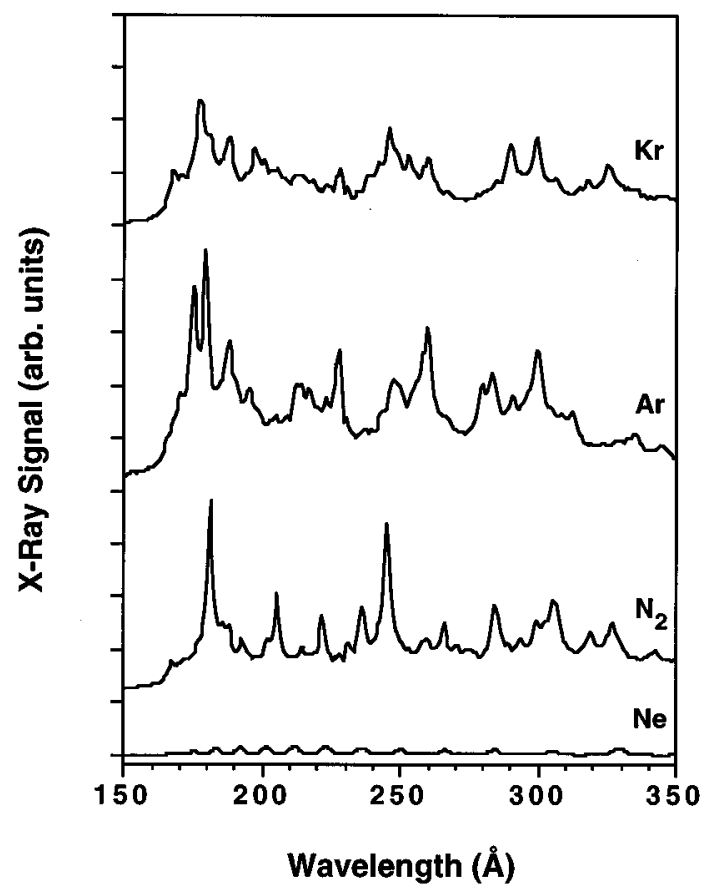

FIG. 17. Time-integrated soft-x-ray emission from $\mathrm{Ne}, \mathrm{N}_{2}, \mathrm{Ar}$, and $\mathrm{Kr}$ with a peak laser intensity of $2 \times 10^{16} \mathrm{~W} / \mathrm{cm}^{2}$ and a gas jet backing pressure of 500 psi. Spectra for $\mathrm{Kr}, \mathrm{Ar}$, and $\mathrm{N}_{2}$ have been offset for clarity.

pare the $\mathrm{N}^{3+} 4 p \rightarrow 2 s$ line at $189 \AA$, the $\mathrm{Ar}^{7+} 4 d \rightarrow 3 p$ line at $179 \AA$, and the strong feature in $\mathrm{Kr}$ at around $175 \AA$ which probably comes from $\mathrm{Kr}^{7+}$. The time resolution of these data

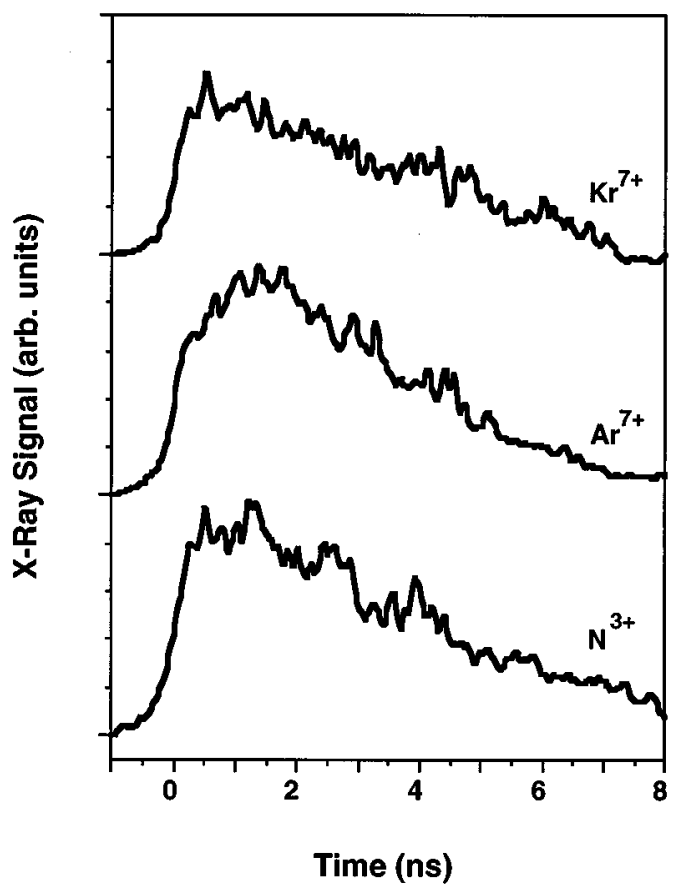

FIG. 18. Time histories of select lines from around $180 \AA$ from the spectral data of Fig. 17. Time histories for Ar and K have been offset for clarity.

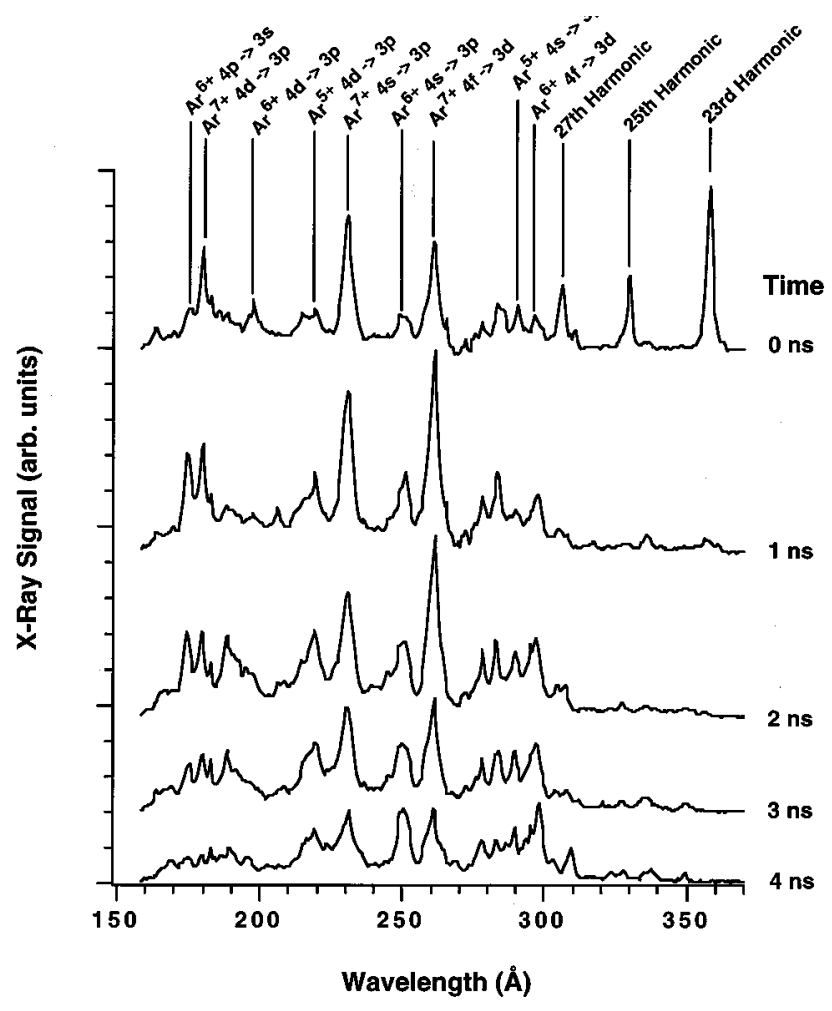

FIG. 19. Spectra of the Ar plasma at intervals of $1 \mathrm{~ns}$ integrated over a time window of $500 \mathrm{ps}$ with a peak intensity of $1 \times 10^{16}$ $\mathrm{W} / \mathrm{cm}^{2}$.

is approximately 280 ps. For all three gases the emission starts promptly after the passage of the laser through the plasma and the decay times are roughly the same for all three species, between 6 and $8 \mathrm{~ns}$. This behavior is qualitatively consistent with our supposition that these high charge states are produced rapidly during the laser pulse inside the cluster. The long decay time is also consistent with the production of a hot plasma. For example, if we assume that the temperature of the plasma is given by the ATI calculation of Fig. 1 $\left(k T_{e} \sim 10 \mathrm{eV}\right)$, the three-body recombination time for $\mathrm{Ar}^{7+}$ according to Eq. (44) is $\sim 100$ ps. This is much more rapid than is observed. These time scales are more consistent with the plasma temperature decay time predicted by the calculations detailed in Sec. VI.

A more quantitative comparison of the soft-x-ray data can be made by comparing the ratio of the various charge states in the plasma as a function of time. Figure 19 shows the spectra of the Ar plasma at intervals of 1 ns integrated over a time window of $500 \mathrm{ps}$ in each case. Initially, the strongest emission comes from the $\mathrm{Ar}^{7+}$ lines. Later, the $\mathrm{Ar}^{6+}$ and $\mathrm{Ar}^{5+}$ lines come up relative to the $\mathrm{Ar}^{7+}$ emission. This confirms the calculation of the cluster ionization under these conditions which indicated that the argon was ionized past the lower charge states with a predominance of Ar charge states of $7+$ and higher. The strong emission from the $\mathrm{Ar}^{7+}$ at the later times probably comes from the recombination of $\mathrm{Ar}^{8+}$ as the plasma cools.

The measured backing pressure dependence of the $\mathrm{Ar}^{7+}$ $4 s \rightarrow 3 p$ line is shown at a peak intensity of $10^{16} \mathrm{~W} / \mathrm{cm}^{2}$ in Fig. 20. The line appears at backing pressures above about $100 \mathrm{psi}$, coinciding with the observed onset of large cluster formation in argon by the Rayleigh scattering measurements. The signal rises rapidly at first and then rolls over at about 


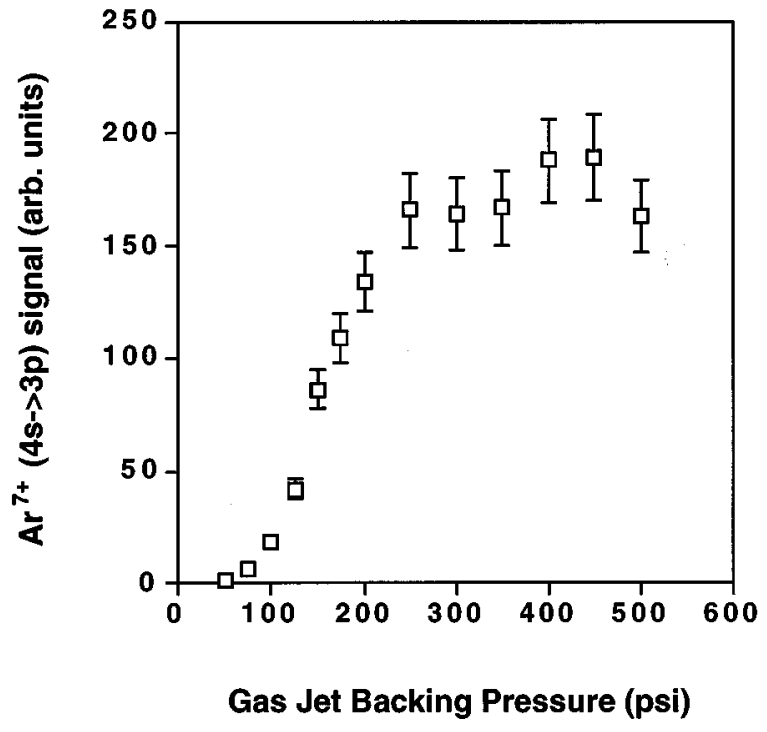

FIG. 20. Measured backing pressure dependence of the $\mathrm{Ar}^{7+}$ $4 s \rightarrow 3 p$ line at a peak intensity of $10^{16} \mathrm{~W} / \mathrm{cm}^{2}$.

$250 \mathrm{psi}$. This rollover point corresponds to an estimated cluster diameter of $60-80 \AA$ based on the estimates of the preceding section and the measured scattered signal at 250 psi. This roll-over may correspond to the onset of cluster production with sizes larger than the optimum size for heating the cluster (see Fig. 9).

\section{HELIUM-CLUSTER MIXTURE EMISSION CHARACTERISTICS}

To more carefully investigate the production of heated electrons by the illumination of the clusters, we conducted a series of experiments in which a small fraction $(0.01-0.1)$ of a gas known to cluster is mixed with $\mathrm{He}$ and passed through the gas jet. Since the He does not itself cluster, observation of the intensity of the Lyman series transitions in $\mathrm{He}^{+}$allowed us to study the dynamics of the bulk plasma without concern for intracluster effects. The presence of the clusters served to absorb laser energy, resulting in a hot thermal plasma which can then collisionally excite the He levels after the clusters expand.

Figure 21 compares the emission spectra of helium for two conditions. The dashed line shows the spectrum when helium alone backs the gas jet. Here the peak laser intensity used is about $3 \times 10^{16} \mathrm{~W} / \mathrm{cm}^{2}$. The intensity required to optically ionize $\mathrm{He}$ to $\mathrm{He}^{2+}$ is approximately $1.5 \times 10^{16} \mathrm{~W} / \mathrm{cm}^{2}$ with a 130-fs laser pulse. Above this intensity Ly- $\alpha$ light will be emitted due to the recombination of electrons into the upper levels of the doubly ionized He. Since no clusters are present, a small amount of signal is observed on the He Ly- $\alpha$ line resulting from this recombination. The solid line in Fig. 21 , however, shows the spectrum of the helium when the backing gas contained 1\% Ar mixed with the helium. Though the peak intensity is only $3 \times 10^{15} \mathrm{~W} / \mathrm{cm}^{2}$, below the threshold for optically ionizing the helium to the required charge state for recombination, the spectrum exhibits strong emission on the Ly- $\alpha$ line as well as the $n=3 \rightarrow 1$ and $4 \rightarrow 1$ transitions. This represents evidence for the presence of

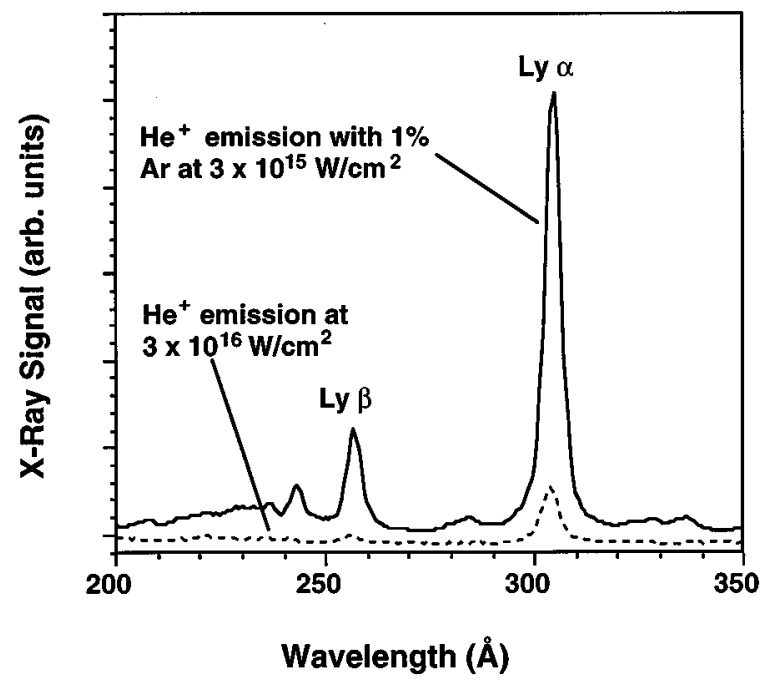

FIG. 21. Emission spectrum with a peak irradiance of $3 \times 10^{16}$ $\mathrm{W} / \mathrm{cm}^{2}$ in pure helium (dashed line) compared with the emission spectrum from a $1 \%$ Ar to $99 \%$ He mixture irradiated with an intensity of $3 \times 10^{15} \mathrm{~W} / \mathrm{cm}^{2}$.

plasma temperatures that are sufficient to collisionally ionize the helium to the doubly ionized state.

In Fig. 22 the time-integrated yield on the Ly- $\alpha$ line in $\mathrm{He}$ is plotted as a function of intensity for a variety of gas mixtures. When a plasma is formed from $100 \%$ pure helium we observe a small amount of Ly- $\alpha$ light at peak intensities above which the tunneling ionization to $\mathrm{He}^{2+}$ begins. Addi-

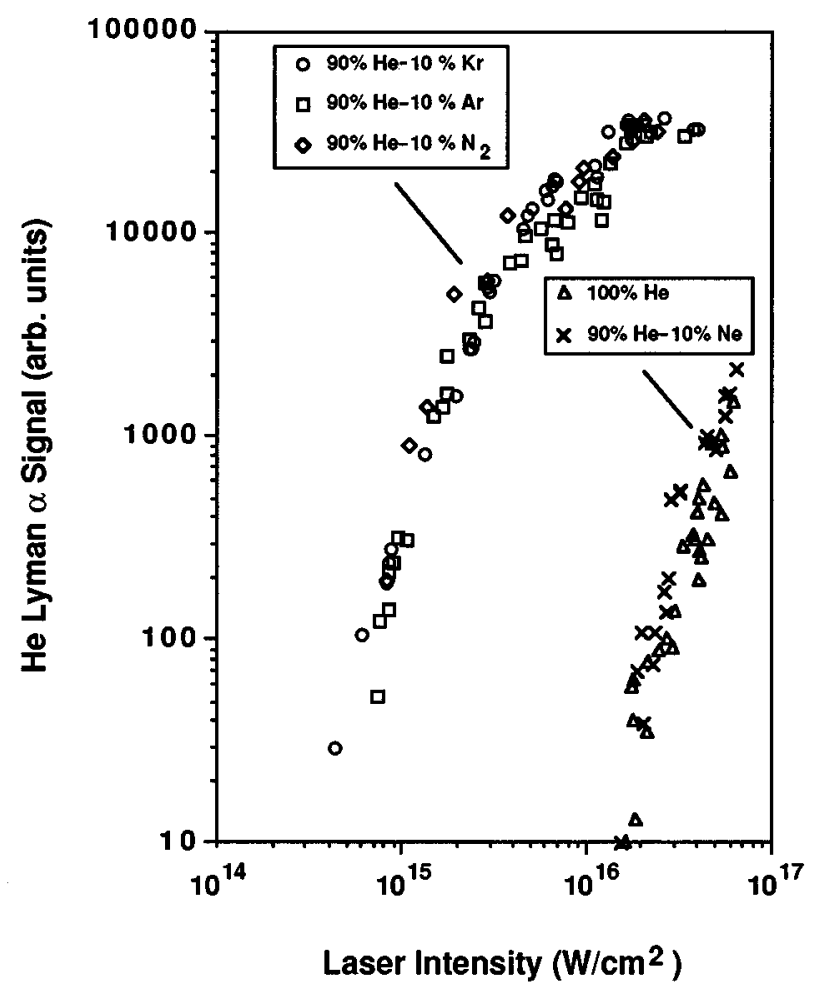

FIG. 22. Integrated yield on the Ly- $\alpha$ line in He plotted as a function of intensity for a variety of gas mixtures. The gas jet backing pressure was 300 psi for all mixtures. 
(a)

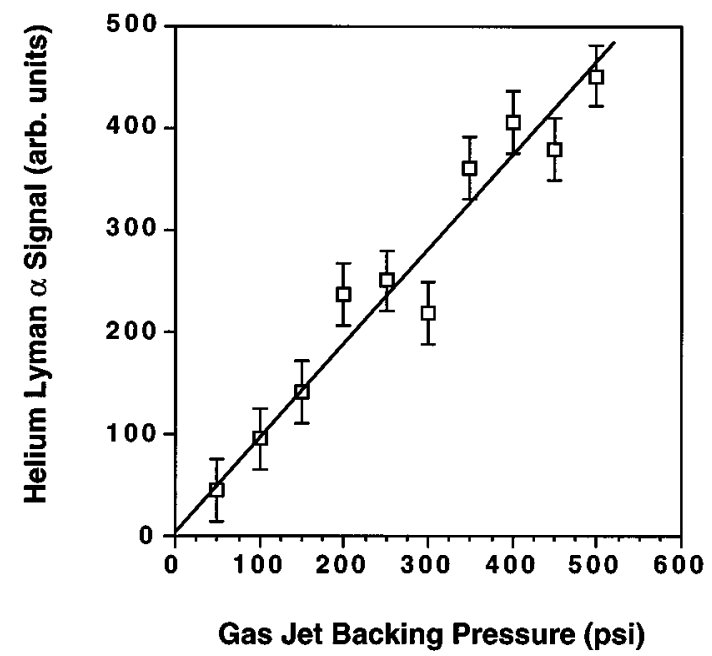

(b)

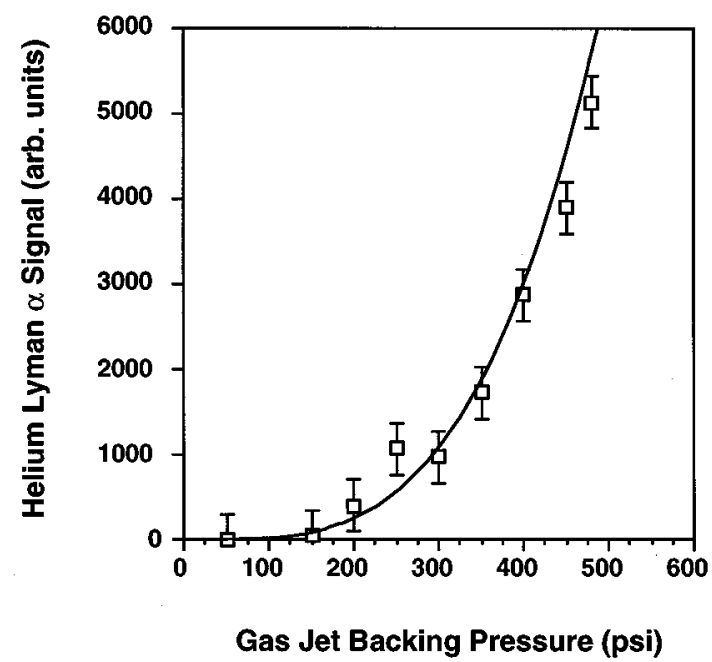

FIG. 23. Ly- $\alpha$ signal emitted as a function of backing pressure for (a) an intensity of $3 \times 10^{16} \mathrm{~W} / \mathrm{cm}^{2}$ in pure $\mathrm{He}$, and (b) an intensity of $3 \times 10^{15} \mathrm{~W} / \mathrm{cm}^{2}$ in a mixture of $1 \% \mathrm{Ar}$ and $99 \% \mathrm{He}$.

tion of a small fraction of $\mathrm{Ne}$ does not significantly change the observed Ly- $\alpha$ signal. This is consistent with the fact that large clusters are absent in the $\mathrm{He}$ and $\mathrm{He} / \mathrm{Ne}$ expansions and the small observed signal is due only to direct strong-field ionization by the laser. When a small amount (10\%) of Ar, $\mathrm{Kr}$, or $\mathrm{N}_{2}$ is mixed with the helium, all gases with a strong propensity for forming large clusters, the magnitude of the He Ly- $\alpha$ signal is significantly enhanced, exceeding the signal of the pure helium by nearly a factor of 100 at the highest intensities. Furthermore, the Ly- $\alpha$ signal appears at an intensity that is 20 times lower than the threshold for the production of $\mathrm{He}^{2+}$ predicted by tunneling ionization.

The importance of the clusters is confirmed when we compare the Ly- $\alpha$ signal dependencies with gas jet backing pressure for the situations when a dopant gas is added to the helium and when impurity is absent. The Ly- $\alpha$ signal emitted as a function of backing pressure for an intensity of $3 \times 10^{16}$ $\mathrm{W} / \mathrm{cm}^{2}$ when no clustering impurity gas is present in the helium is plotted in Fig. 23(a), illustrating the linear increase in signal that is expected due to the linear increase in the number of emitters in the focal volume. The Ly- $\alpha$ signal exhibits a nonlinear increase when $1 \% \mathrm{Ar}$ is present in the

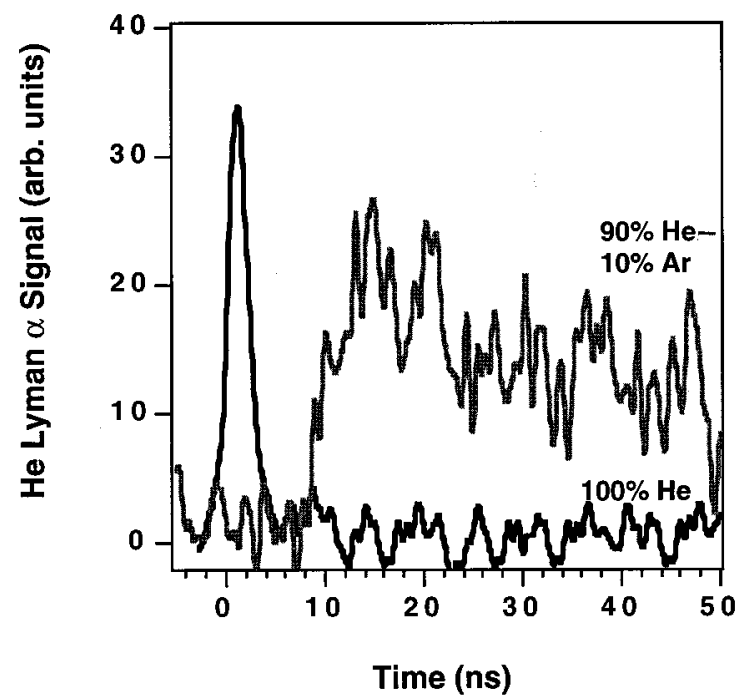

FIG. 24. He Lyman- $\alpha$ time history for an intensity of $7 \times 10^{16}$ $\mathrm{W} / \mathrm{cm}^{2}$ in pure $\mathrm{He}$ (black line), and an intensity of $6 \times 10^{15} \mathrm{~W} / \mathrm{cm}^{2}$ in a mixture of $10 \% \mathrm{Ar}$ and $90 \% \mathrm{He}$ (gray line).

helium jet that is irradiated by a peak intensity of $3 \times 10^{15}$ $\mathrm{W} / \mathrm{cm}^{2}$. The signal increases roughly as $\sim p^{3.2}$. This nonlinear increase in signal is probably due to two factors. The production of the Ly- $\alpha$ radiation is by collisional mechanisms, and is therefore nonlinearly dependent on the density of the gas. This is different than the production of the charge states in the clusters themselves where the collisional mechanisms occur within the solid density of the cluster, a density which is, of course, independent of backing pressure. The rapid rise in Ly $\alpha$ may also be due to the increase of absorption efficiency of the Ar clusters as they grow in size with increasing backing pressure.

The difference in the plasma temperature between the optically ionized (pure $\mathrm{He}$ ) case and the cluster-heated case is most dramatically evident in the comparison of the Ly- $\alpha$ time decay dynamics. The measured time histories over the first $50 \mathrm{~ns}$ after passage by the pulse for both cases are shown in Fig. 24. (The time the laser passes through though the gas jet can be determined by the appearance of the harmonics for these data.) These plots represent an average over ten laser shots. The optically ionized helium $\left(100 \%\right.$ He $I=7 \times 10^{16}$ $\left.\mathrm{W} / \mathrm{cm}^{2}\right)$ exhibits emission immediately $(<1 \mathrm{~ns})$ after creation by the laser followed by a fast $(\sim 2 \mathrm{~ns})$ fall. The plasma heated by the presence of clusters $\left(10 \%\right.$ Ar $I=6 \times 10^{15}$ $\mathrm{W} / \mathrm{cm}^{2}$ ) shows no Ly- $\alpha$ signal immediately after the laser, due to the fact that the intensity of the laser is sufficient to ionize to $\mathrm{He}^{+}$only. The hot electrons from the clusters, however, serve to collisionally ionize the He on a long $(\sim 10 \mathrm{~ns})$ time scale resulting in Ly- $\alpha$ emission on a $\sim 100-n s$ time scale. The slow turn on of the He line is because of the slow rate of collisional ionization to $\mathrm{He}^{2+}$ in the relatively low density of the helium plasma.

A calculation of the time scale for the population of the $n=2$ state in $\mathrm{He}^{+}$using the calculated hydrodynamic temperature history in FLY is shown in Fig. 25 for the first $15 \mathrm{~ns}$. The solid curve assumes the ATI prediction for the plasma temperature of $40 \mathrm{eV}$ and an initial population of $100 \% \mathrm{He}^{2+}$ 


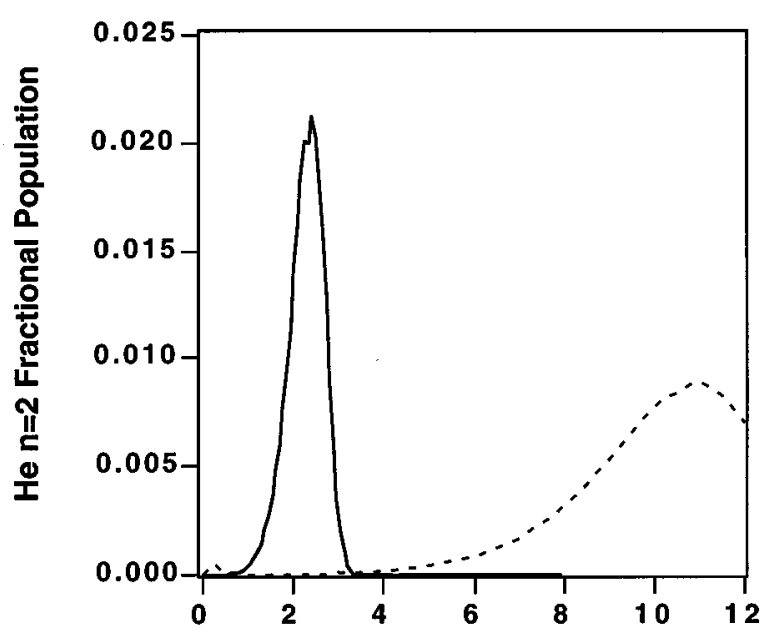

Time (ns)

FIG. 25. Calculation of the time scale for the population of the $n=2$ state in $\mathrm{He}^{+}$for (a) an ATI heated plasma $(35 \mathrm{eV})$ and initial stripped $\mathrm{He}^{2+}$ ions, and (b) a cluster-heated plasma $(250 \mathrm{eV})$ with $\mathrm{He}$ initially stripped to $\mathrm{He}^{+}$.

produced through optical ionization. The calculation reproduces the observed time scale of decay for this case. The dashed line in Fig. 24 shows the $n=2$ population for a plasma with an initial temperature of $250 \mathrm{eV}$. The calculation shows that the time scale for population of this level through collisional ionization is roughly $10 \mathrm{~ns}$, in excellent agreement with the observed data. The population of this level in helium in this case is predominantly by collisional ionization of the $\mathrm{He}^{+}$followed by three-body recombination. Since the Ar clusters contribute only $\sim 30 \%$ of the electrons in the plasma, this modeling indicates that the cluster electrons probably have an initial temperature of the order of $500-1000 \mathrm{eV}$.

\section{X-RAY EMISSION IN Kr BELOW 100 Å}

The enhanced absorption by clusters also suggests that short wavelength $(<100 \AA)$ x rays may be produced at relatively modest laser intensity. Figure 26 shows the timeintegrated spectrum between 40 and $100 \AA$ produced by focusing the laser to an intensity of approximately $1.5 \times 10^{16}$ $\mathrm{W} / \mathrm{cm}^{2}$ into the gas jet backed by 500 psi of pure Kr. The spectrum exhibits strong emission from the $4 p-3 d$, and $4 s$ $3 p$ arrays of $\mathrm{Kr}^{10+}, \mathrm{Kr}^{11+}, \mathrm{Kr}^{12+}$, and $\mathrm{Kr}^{13+}$ [54,55]. Tunnel ionization predicts that intensities of $3,4,6$, and $8 \times 10^{17}$ $\mathrm{W} / \mathrm{cm}^{2}$, respectively, are required to produce these states by optical ionization, over an order of magnitude in excess of the actual laser intensity used here. The observed charge states in $\mathrm{Kr}$ agree very well with the predicted ionization stages of the cluster calculations of Fig. 11(b) which shows that under these intensities, ionization in the cluster should produce $\mathrm{Kr}$ ionized to $11-14+$.

Similar to the softer-X-ray emission of $\mathrm{Kr}$ when large clusters are present in the gas jet target, the emission from these lines is long lived. The time history of the $\mathrm{Kr}^{10+}$ $4 p \rightarrow 3 d$ line is shown in Fig. 27; the streak camera temporal

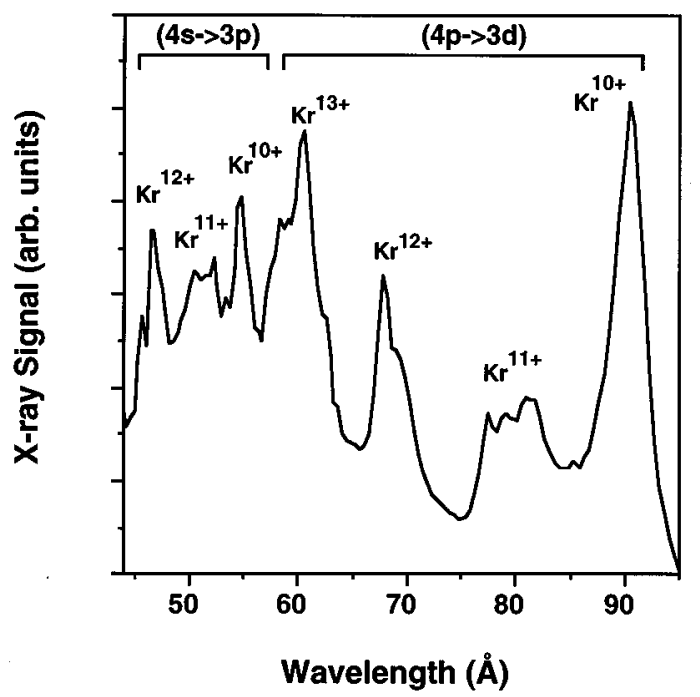

FIG. 26. Time-integrated spectrum in $\mathrm{Kr}$ with an intensity of $1.5 \times 10^{16} \mathrm{~W} / \mathrm{cm}^{2}$ into the gas jet backed by $500 \mathrm{psi}$.

resolution of these data is approximately $280 \mathrm{ps}$. The radiation from this line is emitted for nearly $3 \mathrm{~ns}$ after the laser produces the plasma. The long lifetime is consistent with the long-lived emission of a hot, underdense bulk plasma. The actual lifetime of these lines is insensitive to the gas jet backing pressure. The measured decay time of the $\mathrm{Kr}^{10+} 4 p \rightarrow 3 d$ line is plotted as a function of backing pressure for a peak intensity of $1 \times 10^{16} \mathrm{~W} / \mathrm{cm}^{2}$ (defined as the time for the signal to fall to $1 / e$ of its peak value) in Fig. 28. The decay time is roughly 2 ns over the range of backing pressures from 200 to 700 psi. These data suggest that the dominant mechanism for the production of this radiation is collisional excitation. The time scale for collisional excitation will be set by the cooling rate of the plasma which is largely determined by the speed of the hydrodynamic expansion. This speed, roughly given by the sound speed of the plasma, is independent of the gas density. If the time scale were set by the rate of recombina-

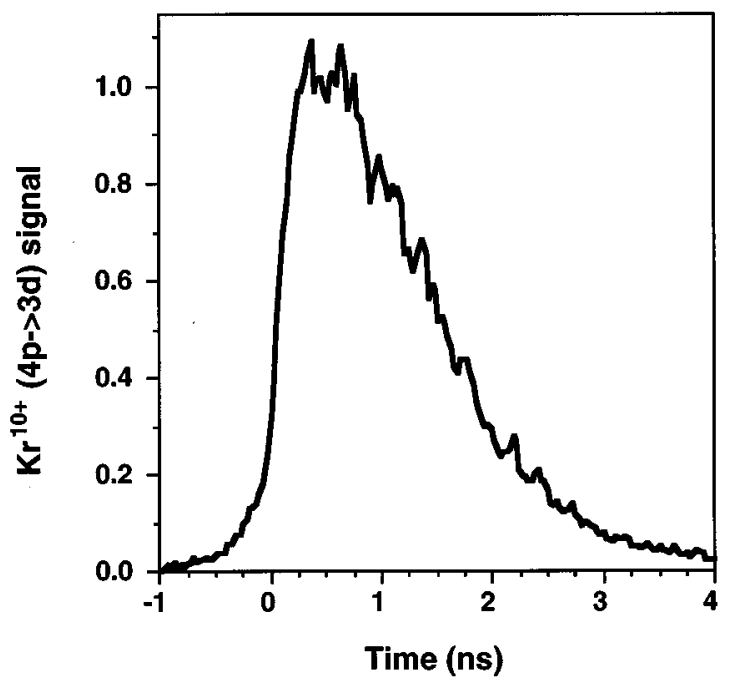

FIG. 27. Time history of the $\mathrm{Kr}^{10+} 4 p \rightarrow 3 d$ line. Streak camera resolution is $280 \mathrm{ps}$. 


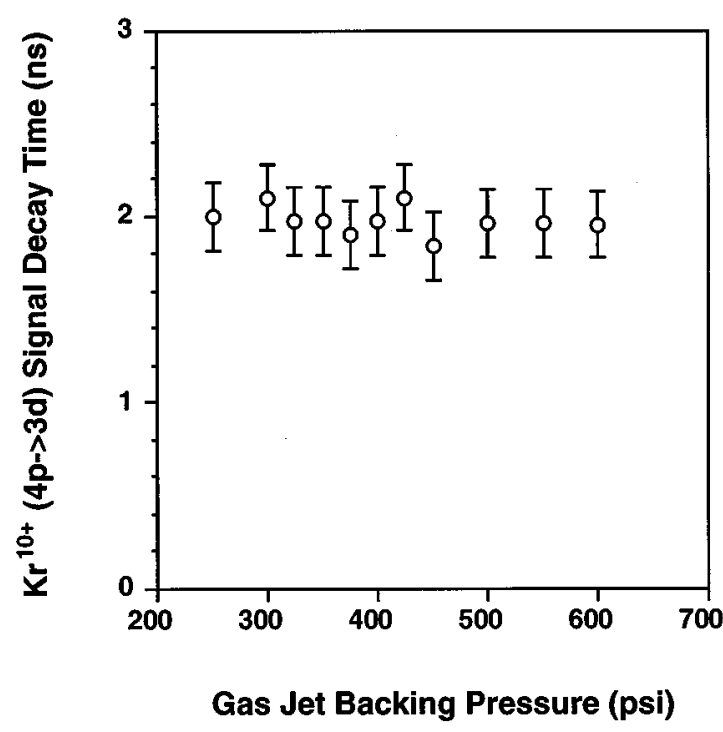

FIG. 28. Measured decay time of the $\mathrm{Kr}^{10+} 4 p \rightarrow 3 d$ line is plotted as a function of backing pressure for a peak intensity of $1 \times 10^{16} \mathrm{~W} / \mathrm{cm}^{2}$.

tion [Eq. (44)] the decay time would be strongly dependent on the gas density.

Evidence for the interaction of the laser with solid density clusters is found when the time history of the $\mathrm{Kr}$ emission is observed over the first 100 ps. Data showing the streaked spectrum of $\mathrm{Kr}$ are shown in Fig. 29(a). The time resolution of these data is roughly 20 ps. Figure 29(b) shows a lineout illustrating the time history of the $\mathrm{Kr}^{10+} 90-\AA$ line for the first 100 ps after illumination by the laser (here the time resolution has been decreased to $10 \mathrm{ps}$ ). We observe an initial spike in the emission of the $\mathrm{Kr}^{10+}$ line, faster than the time resolution of the streak camera, followed by the longlived emission demonstrated in Fig. 27. A similar spike is seen on all the charge states lines as well as in the background continuum emission. This initial spike is indicative of intense $\mathrm{x}$-ray emission by the dense cluster microplasmas immediately after heating by the laser. The fast $(<1 \mathrm{ps})$ expansion of the cluster is then followed by lower-intensity emission by the low-density bulk plasma on a long ( $3 \mathrm{~ns}$ ) time scale. The fast rise time of the emission from the high charge states indicates that rapid ionization $(<1 \mathrm{ps})$ occurs to $\mathrm{Kr}^{10+}-\mathrm{Kr}^{13+}$ within the cluster, consistent with our calculations. Though the number of photons contained within this spike is much less than the total number of photons that are emitted by the plasma, the relative brightness is higher than the long-lived emission. If the emission is only a few hundred fs in duration, as our model suggests, then the relative brightness of the spike would be two orders of magnitude above that of the underdense plasma emission.

\section{SHORT WAVELENGTH EMISSION IN Ar}

Short wavelength emission is observed under similar conditions in Ar as well. A time-integrated spectrum of Ar emission from 20 to $140 \AA$ is shown in Fig. 30. The gas jet backing pressure was $450 \mathrm{psi}$ and the laser was focused to $8 \times 10^{15} \mathrm{~W} / \mathrm{cm}^{2}$. The most dramatic aspect of this spectrum is the observation of strong emission from lines at wavelengths
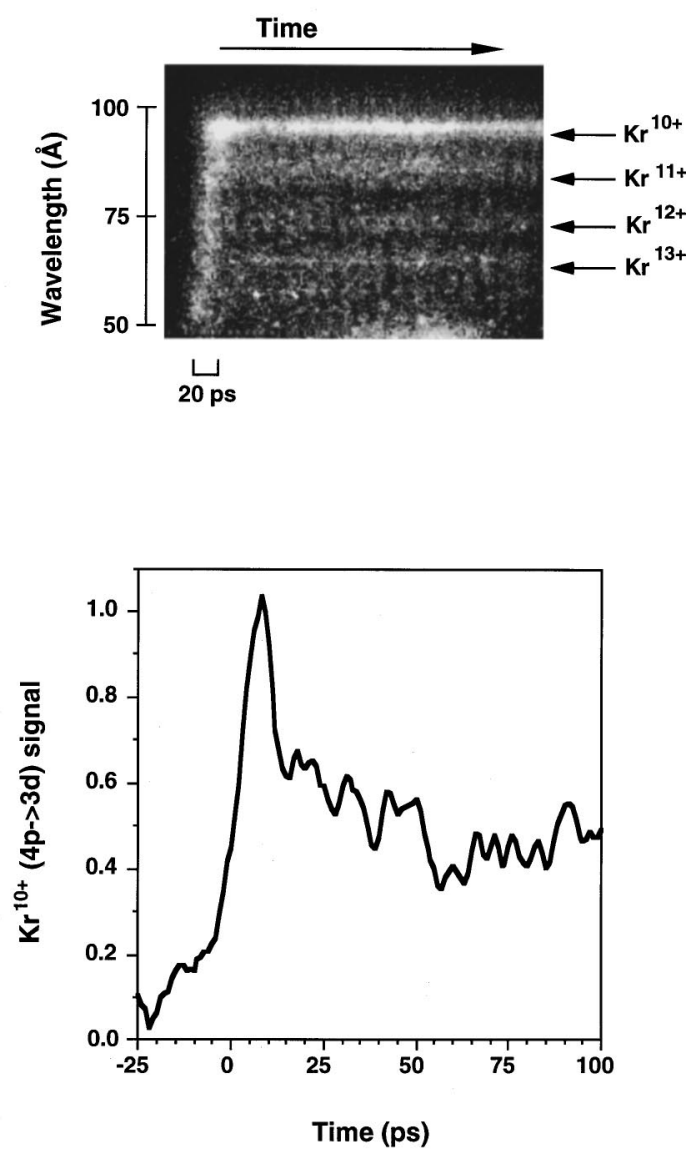

FIG. 29. (a) Streaked spectrum of $\mathrm{Kr}$ with a time resolution of 20 ps. (b) Time history of the $\mathrm{Kr}^{10+} 90-\AA$ line for the first 100 ps (with $\sim 10$-ps time resolution).

below $50 \AA$ in neonlike $\mathrm{Ar}\left(\mathrm{Ar}^{8+}\right)$ [56]. We estimate that the photon yield on these lines indicates that up to $1-5 \%$ of the laser energy is converted to $\mathrm{x}$ rays in this wavelength range. To produce this charge state by tunnel ionization would require a focused intensity of $>1.5 \times 10^{18} \mathrm{~W} / \mathrm{cm}^{2}$, more than

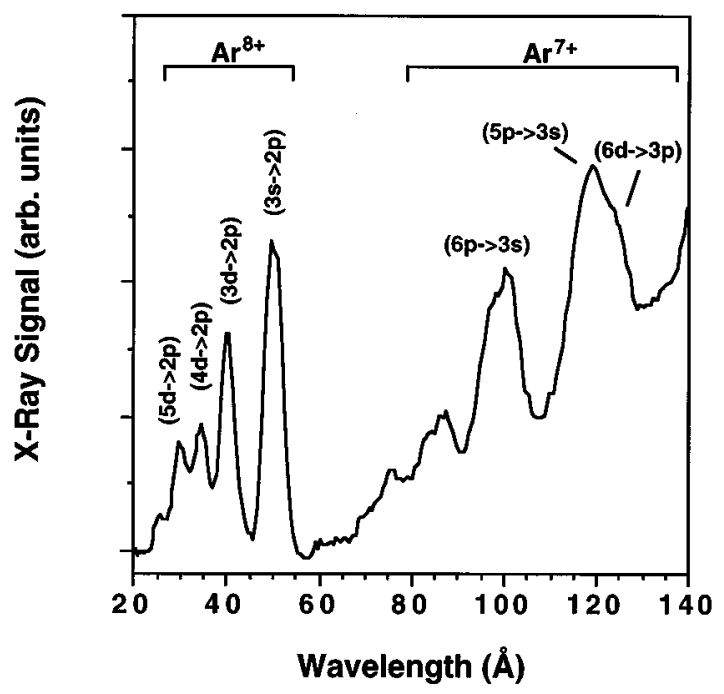

FIG. 30. Time-integrated spectrum of Ar emission from 20 to $140 \AA$ with a gas jet backing pressure of $450 \mathrm{psi}$ and an intensity of $8 \times 10^{15} \mathrm{~W} / \mathrm{cm}^{2}$. 


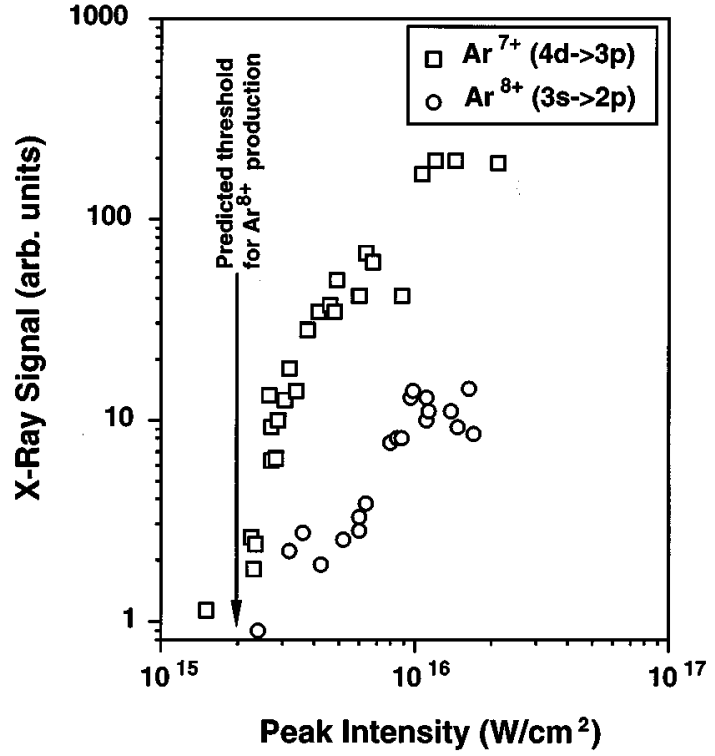

FIG. 31. Measured signal of the $\mathrm{Ar}^{8+} 3 s \rightarrow 2 p$ line and the $\mathrm{Ar}^{7+}$ $4 d \rightarrow 3 p$ line as a function of intensity.

two orders of magnitude higher than that used in the experiment. In fact, the appearance intensity of this charge state is consistent with the predictions of the calculation illustrated in Fig. 11. The measured signals of the $\mathrm{Ar}^{8+} 3 s \rightarrow 2 p$ line and the $\mathrm{Ar}^{7+} 4 d \rightarrow 3 p$ line are plotted versus intensity in Fig. 31 . The appearance of these lines at an intensity of $2 \times 10^{15}$ $\mathrm{W} / \mathrm{cm}^{2}$ for the $\mathrm{Ar}^{7+}$ and $3 \times 10^{15} \mathrm{~W} / \mathrm{cm}^{2}$ for the $\mathrm{Ar}^{8+}$ signal agree very well with the calculation of Ar ionization in the cluster, whose onset we have illustrated in Fig. 31.

The time history of the neonlike Ar emission is of a duration that is comparable to the $\mathrm{Kr}$ emission. Figure 32 illustrates the characteristic emission history of the $\mathrm{Ar}^{8+} 3 s \rightarrow 2 p$ line at $49 \AA$. The signal exhibits a $\sim 1$-ns drop followed by a longer $(\sim 4 \mathrm{~ns})$ tail. We have modeled the dynamics of the Ar emission in detail to describe this history. Figure 33 shows the calculated time histories of the temperature at various radial points (as before, we have used the calculated temperature as a function of intensity to determine the initial

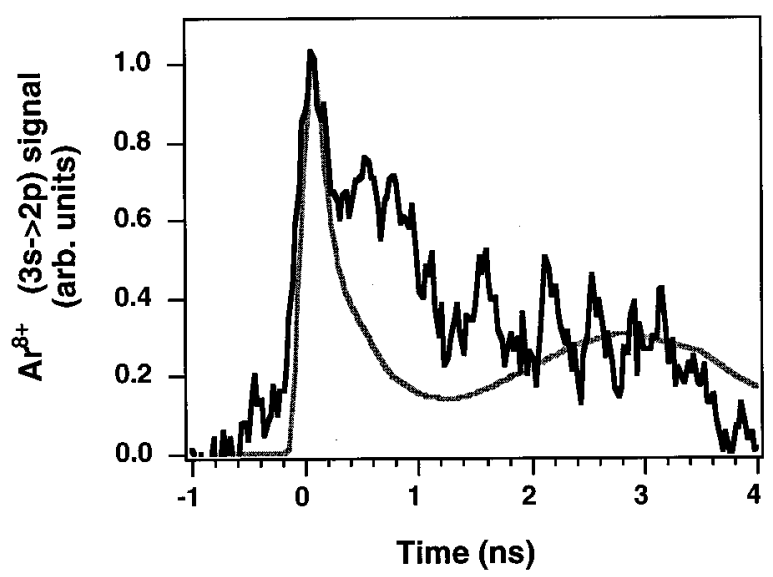

FIG. 32. Measured (solid line) and calculated (gray line) emission history of the $\mathrm{Ar}^{8+} 3 s \rightarrow 2 p$ line at $49 \AA$.

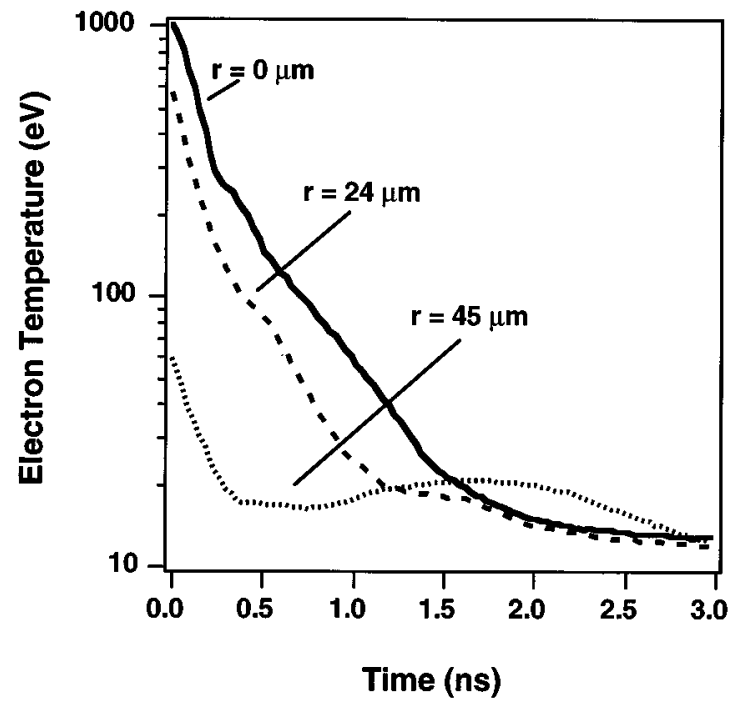

FIG. 33. Calculated time history of the temperature in an $\mathrm{Ar}$ plasma at various radial points in the laser focus.

temperature profile). We have assumed that the peak initial temperature is $1000 \mathrm{eV}$. The temperature drops rapidly at first due to the free streaming cooling to the cold plasma that surrounds the hot plasma at the center of the laser focus. This is followed by a slower decay from $\sim 100-200 \mathrm{eV}$ by hydrodynamic expansion. To calculate the Ar emission time history we have calculated the charge state time histories at various radial points using FLY.

The population of the $3 s \rightarrow 2 p$ transition was estimated by assuming pumping by collisional excitation in the $\mathrm{Ar}^{8+}$ ions and recombination pumping from $\mathrm{Ar}^{9+}$. The collisional excitation rates of Mohan, Dourneuf, and Hibbert were used to calculate the magnitude of the first mechanism [57]. The recombination rate of Eq. (44) was used to determine the rate at which the upper levels of $\mathrm{Ar}^{9+}$ are filled. We then assume that these electrons cascade through the lower levels and populate the $n=3$ levels by their statistical weights. The calculated time history of the $\operatorname{Ar} 3 s \rightarrow 2 p$ emission found by using these assumptions is shown as a gray line in Fig. 32. The large initial signal over the first $\sim 1$ ns results from collisional excitation. The longer tail comes from the recombination of $\mathrm{Ar}^{9+}$. The combination of these mechanisms seems to adequately explain the emission time history. The fall of the collisional contribution to the signal is faster compared to the $\mathrm{Kr}$ time history due to the higher energy of the transition and the faster expansion rate of the Ar plasma (since the $\mathrm{Kr}$ ions are more massive than the Ar ions).

\section{PREPULSE EXPERIMENTS}

Since the clusters expand rapidly after heating by the laser, the presence of a laser prepulse of sufficient energy to ionize the cluster should be sufficient to destroy the clusters. To explore this effect we examined the yields and time history of a cluster plasma with and without a laser prepulse. In the first experiment we generated a prepulse by placing a fused silica blank in the beam with a hole removed in the center of the beam. The part of the beam that passed though the hole preceded the main part of the beam by roughly 35 


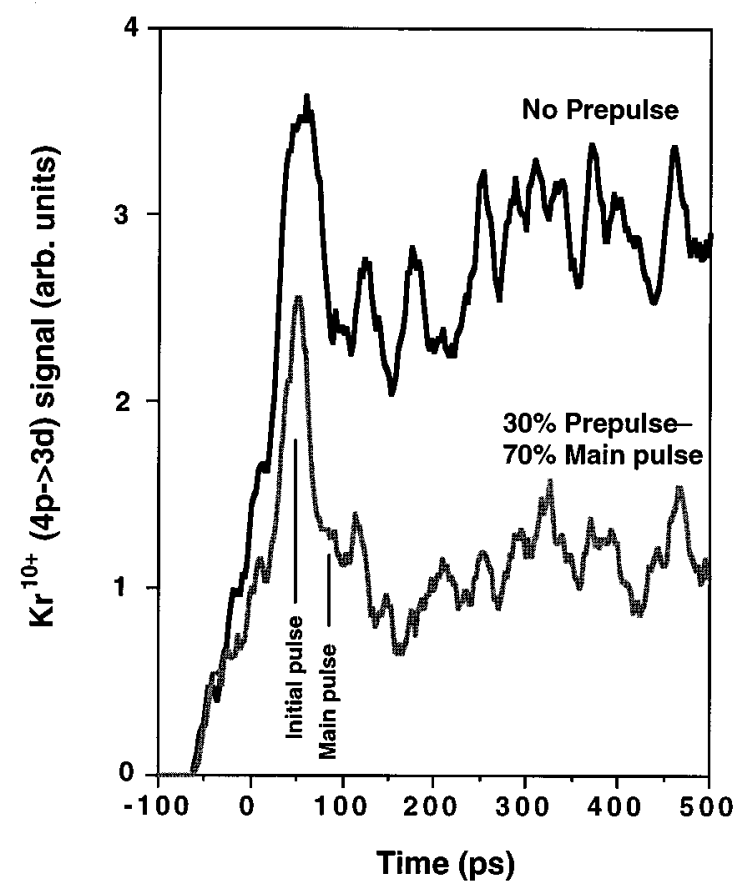

FIG. 34. Fast time history of the $\mathrm{Kr}^{10+}$ emission for a peak intensity of $2 \times 10^{16} \mathrm{~W} / \mathrm{cm}^{2}$ when no prepulse is used (dark line) compared to the case when a $30 \%$ prepulse was allowed into the chamber 35 ps before the main pulse (gray line).

ps. The intensity of this pulse was about $30 \%$ of the main pulse.

The fast time history of the $\mathrm{Kr}^{10+}$ emission for a peak intensity of $2 \times 10^{16} \mathrm{~W} / \mathrm{cm}^{2}$ is shown in Fig. 34 (dark line). This is compared to the case when both the prepulse and the main pulse were focused into the chamber (gray line). The case in which both pulses were focused into the gas exhibit only one spike in the $\mathrm{x}$-ray emission, though two pulses traversed the jet. Furthermore, the $x$-ray yield of the case when the prepulse is present is approximately 0.3 times that of the case in which only the main pulse was focused into the jet. When the prepulse was present, the signal is reduced because only the prepulse energy is absorbed. The main pulse traverses underdense plasma only and does not deposit any substantial energy in the plasma. Therefore no spike in $\mathrm{x}$ rays is observed coincident with the main pulse in this case.

To more accurately determine the intensity at which the clusters disassemble, a second experiment was performed in which a small prepulse 14 ns before the main pulse is generated by allowing some pulse energy to leak out of the regenerative amplifier cavity. Tuning the timing of the Pockels cell in the cavity permitted control over the amplitude of the prepulse which was monitored on a fast photodiode and a sampling scope. Figure 35 illustrates the dramatic difference in the x-ray spectrum between the case when Ar is irradiated with an intensity of $2 \times 10^{16} \mathrm{~W} / \mathrm{cm}^{2}$ and no prepulse is present and when a prepulse of approximately $8 \times 10^{14}$ $\mathrm{W} / \mathrm{cm}^{2}$ precedes the main pulse. The strong line and continuum emission produced upon irradiation of $\mathrm{Ar}$ in the gas jet disappears completely when the prepulse is added. Only the harmonics, which are generated by individual atoms, are observable when the prepulse is present.

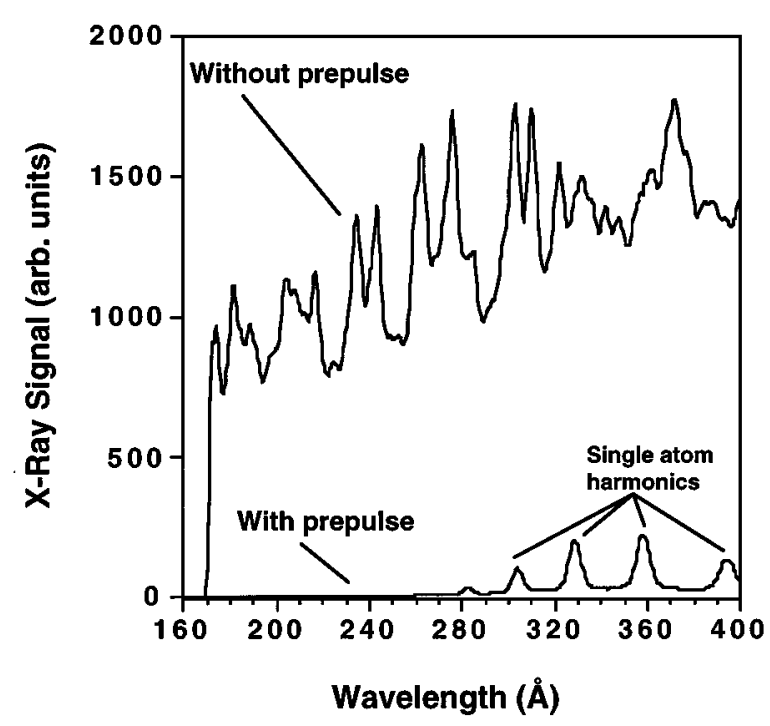

FIG. 35. Comparison of time-integrated emission from Ar when irradiated with an intensity of $2 \times 10^{16} \mathrm{~W} / \mathrm{cm}^{2}$ and no prepulse is present and when a prepulse of approximately $8 \times 10^{14} \mathrm{~W} / \mathrm{cm}^{2}$ precedes the main pulse by $14 \mathrm{~ns}$.

A similar effect can be seen when a mixture of $\mathrm{He}$ and $10 \% \mathrm{Ar}$ is irradiated. Figure 36 shows the helium spectrum with a peak intensity of $7 \times 10^{15} \mathrm{~W} / \mathrm{cm}^{2}$ in the main pulse. Addition of a prepulse of $1 \times 10^{15} \mathrm{~W} / \mathrm{cm}^{2}$ effectively lowers the Ly- $\alpha$ emission to unobservable levels. The threshold for the disassembly of the clusters begins abruptly with increasing prepulse intensity. The He Ly- $\alpha$ yield for a fixed main pulse intensity of $7 \times 10^{15} \mathrm{~W} / \mathrm{cm}^{2}$ is shown as a function of prepulse intensity in Fig. 37. The helium signal drops rapidly for prepulse intensities above about $4 \times 10^{14} \mathrm{~W} / \mathrm{cm}^{2}$. This sharp drop is probably due to the nonlinear nature of the tunnel ionization rate for the Ar atoms in the cluster.

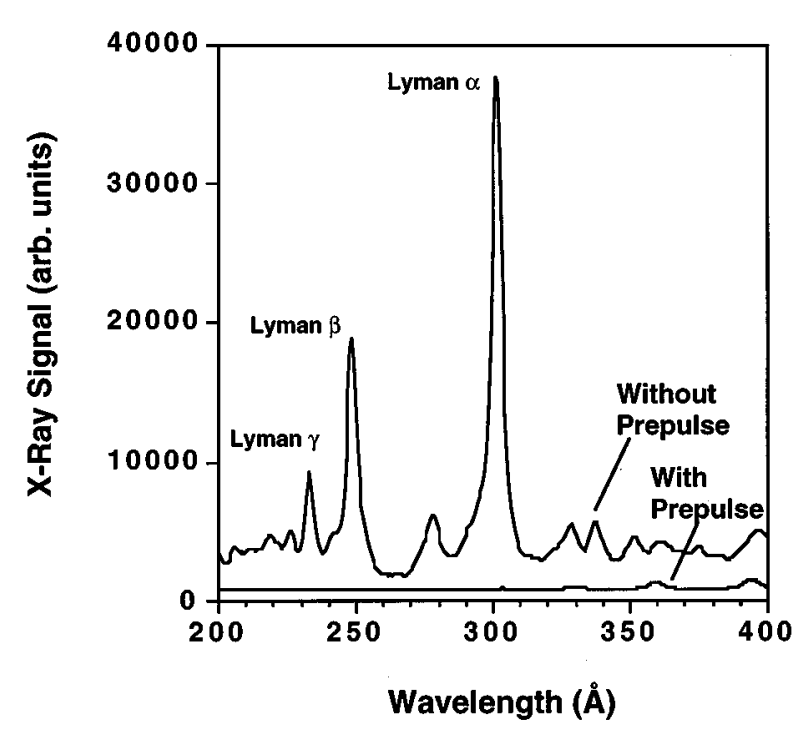

FIG. 36. Helium emission spectrum with a $10 \%$ Ar mixture with a peak intensity of $7 \times 10^{15} \mathrm{~W} / \mathrm{cm}^{2}$ in the main pulse with and without a $14-\mathrm{ns}$ prepulse of $1 \times 10^{15} \mathrm{~W} / \mathrm{cm}^{2}$. 


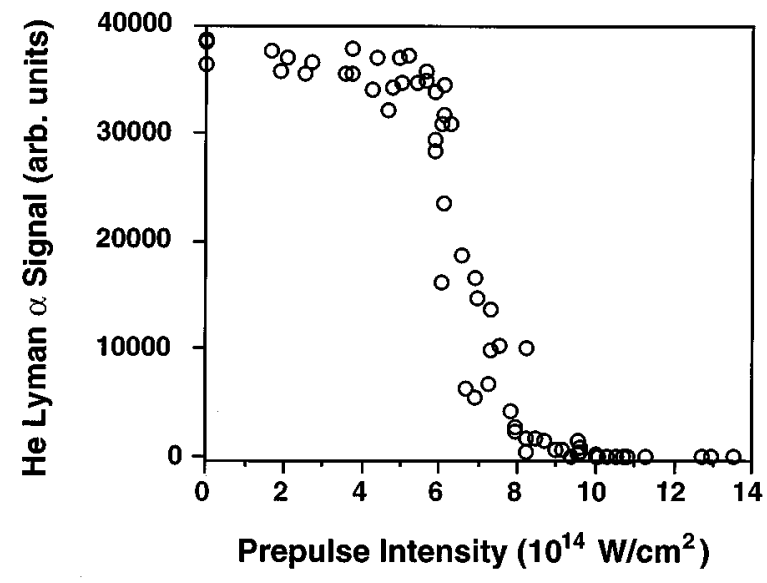

FIG. 37. He Ly- $\alpha$ yield with a $10 \%$ Ar mixture for a fixed main pulse intensity of $7 \times 10^{15} \mathrm{~W} / \mathrm{cm}^{2}$ as a function of 14-ns prepulse intensity.

\section{X-RAY YIELDS FOR LONG AND SHORT PULSES}

The dramatic effect of a prepulse on the plasma emission from a gas containing clusters suggests that there should be a very large difference in the $\mathrm{x}$-ray yields between when a long $(\sim 1 \mathrm{~ns})$ and a short $(\sim 100 \mathrm{fs})$ pulse is used. Since the clusters disassemble on a $<1$-ps time scale, the short pulse interacts with the solid density particles throughout the pulse envelope. The long pulse, however, essentially interacts with underdense plasma through the majority of the pulse. The use of the long pulse focused into the jet will start standard avalanche breakdown in the gas and will heat the lowdensity gas by collisional absorption.

To illustrate this point, we measured the x-ray yield in $\mathrm{Ar}$ in the wavelength band between 300 and $170 \AA$ for cases of short-pulse and long-pulse illumination (Fig. 38). We estimated the $\mathrm{x}$-ray yield by comparing the signal level with

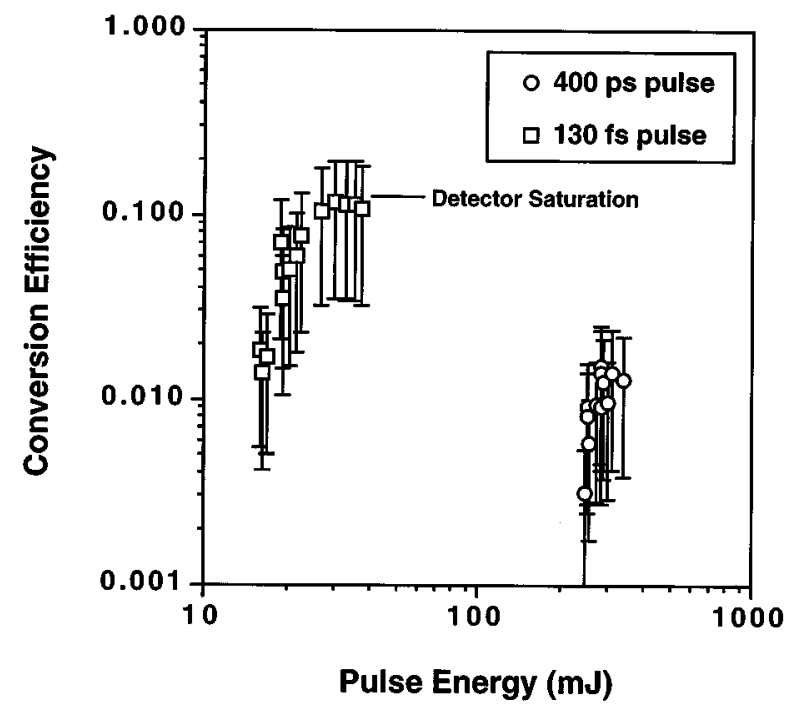

FIG. 38. X-ray yield in Ar in the wavelength band between 300 and $170 \AA$ for cases of short-pulse (130 fs) and long-pulse (400 ps) illumination. harmonics yields under known conversion efficiency conditions [58]. We also estimated the yield from the known throughput of the spectrometer and the gain of the microchannel plate to confirm that this was consistent with the numbers arrived at through comparison with the harmonics. The long pulse for this experiment was derived by simply focusing the uncompressed LiSAF pulse, which has a 400-ps width, into the chamber. The $\mathrm{x}$-ray conversion efficiency is plotted for the long- and short-pulse cases in Fig. 38. For the case of the long pulse, $>0.25 \mathrm{~J}$ of energy was required $\left(I \sim 3 \times 10^{13} \mathrm{~W} / \mathrm{cm}^{2}\right)$ to achieve any significant signal in this wavelength range. The conversion efficiency for this case reached just above $1 \%$ for approximately $0.5 \mathrm{~J}$ of energy. The short pulse, however, exhibited a much greater conversion efficiency at a much more modest energy. The conversion efficiency increased into the $10 \%$ range for $30-40 \mathrm{~mJ}$ of laser energy. Saturation of the MCP prevented us from increasing the energy still further.

This sort of conversion efficiency is comparable to x-ray conversion efficiencies attainable with flat solid targets [10]. Thus the use of clusters increases the coupling efficiency of the laser light into the target material to a level that is comparable to a solid target. In principle, using an extended source of clusters, such as a gas jet with a slit nozzle, even higher conversion efficiencies may be possible.

\section{CONCLUSION}

In conclusion, we have shown that large clusters produced in expanding gas jets can be used to produce hot, moderatedensity plasmas with intense, short-pulse lasers. We find that the thermal plasmas produced by the illumination of clusters by femtosecond pulses of $10^{16}-10^{17} \mathrm{~W} / \mathrm{cm}^{2}$ dominate the plasma kinetics, producing emission from high charge states that can last for many nanoseconds. Our experimental results indicate that the clusters are, first, rapidly heated during the laser pulse by collisional inverse bremsstrahlung, followed by rapid expansion of the clusters. The hot, underdense plasma that results then emits soft-x-ray radiation over long (ns) time scales due to collisional excitation and three-body recombination.

These experimental results are in good agreement with modeling of the laser-cluster interaction and the dynamics of the underdense plasma that results after the heated clusters expand. These plasmas exhibit dramatically enhanced absorption of the laser light relative to pure gases, with plasma temperatures well in excess of that expected from the illumination of a low-density gas alone. Furthermore, the x-ray yields are comparable to those that can be achieved with solid targets. These cluster-heated plasmas have the potential for providing a source of strong, x-ray radiation with the modest irradiance $\left(10^{15}-10^{17} \mathrm{~W} / \mathrm{cm}^{2}\right)$ produced by smallscale short-pulse lasers through a unique combination of the advantages inherent to both solid and gas targets.

\section{ACKNOWLEDGMENTS}

We would like to acknowledge the help of R. A. Smith and the technical assistance of R. Jones. This work was supported by the AFOSR and conducted under the auspices of the DOE under Contract No. W-7405-Eng-48. 
[1] M. D. Perry and G. Mourou, Science 264, 917 (1994).

[2] A. L'Huillier, L. Lompré, G. Mainfray, and C. Manus, in Atoms in Intense Laser Fields, edited by M. Gavrila (Academic, Boston, 1992), pp. 139-202.

[3] Y. Nagata, K. Midorikawa, S. Kubodera, M. Obara, H. Tashiro, and K. Toyoda, Phys. Rev. Lett. 71, 3774 (1993).

[4] B. E. Lemoff, G. Y. Yin, C. L. Gordon III, C. P. J. Barty, and S. E. Harris, Phys. Rev. Lett. 74, 1574 (1995).

[5] J. D. Kmetec, C. L. Gordon III, J. J. Macklin, B. E. Lemoff, G. S. Brown, and S. E. Harris, Phys. Rev. Lett. 68, 1527 (1992).

[6] A. P. Fews, P. A. Norreys, F. N. Beg, A. R. Bell, A. E. Dangor, C. N. Danson, P. Lee, and S. J. Rose, Phys. Rev. Lett. 73, 1801 (1994).

[7] W. L. Kruer, The Physics of Laser Plasma Interactions (Addison-Wesley, Redwood City, CA, 1988).

[8] M. M. Murnane, H. C. Kapteyn, S. P. Gordon, J. Bokor, E. N. Glytsis, and R. W. Falcone, Appl. Phys. Lett. 62, 1068 (1993).

[9] D. F. Price, R. M. More, R. S. Walling, G. Guethlein, R. L. Shepard, R. E. Stewart, and W. E. White, Phys. Rev. Lett. 75, 252 (1995).

[10] S. P. Gordon, T. Donnelly, A. Sullivan, H. Hamster, and R. W. Falcone, Opt. Lett. 19, 484 (1994).

[11] D. G. Stearns, O. L. Landen, E. M. Campbell, and J. H. Scofield, Phys. Rev. A 37, 1684 (1988).

[12] C. Wülker, W. Theobald, D. Ouw, F. P. Schäfer, and B. N. Chichkov, Opt. Commun. 112, 21 (1994).

[13] O. F. Hagena and W. Obert, J. Chem. Phys. 56, 1793 (1972).

[14] A. McPherson, T. S. Luk, B. D. Thompson, A. B. Borisov, O. B. Shiryaev, X. Chen, K. Boyer, and C. K. Rhodes, Phys. Rev. Lett. 72, 1810 (1994).

[15] A. McPherson, B. D. Thompson, A. B. Borisov, K. Boyer, and C. K. Rhodes, Nature (London) 370, 631 (1994).

[16] T. Ditmire, T. Donnelly, R. W. Falcone, and M. D. Perry, Phys. Rev. Lett. 75, 3122 (1995).

[17] O. F. Hagena, Surf. Sci. 106, 101 (1981).

[18] O. F. Hagena, Rev. Sci. Instrum. 63, 2374 (1992).

[19] V. M. Smirnov, Usp. Fiz. Nauk 164, 665 (1994) [Phys. Usp. 37, 621 (1994)].

[20] J. Wörmer, V. Guzielski, J. Stapelfeldt, and T. Möller, Chem. Phys. Lett. 159, 321 (1989).

[21] J. Farges, M. F. d. Feraudy, B. Raoult, and G. Torchet, J. Chem. Phys. 84, 3491 (1986).

[22] O. Abraham, J. H. Binn, B. G. Deboer, and G. D. Stein, Phys. Fluids 24, 1017 (1981).

[23] C. L. Briant and J. J. Burton, J. Chem. Phys. 63, 2045 (1975).

[24] N. H. Burnett and P. B. Corkum, J. Opt. Soc. Am. B 6, 1195 (1989).

[25] M. V. Ammosov, N. B. Delone, and V. P. Krainov, Zh. Eksp. Teor. Fiz. 91, 2008 (1986) [Sov. Phys. JETP 64, 1191 (1986)].

[26] T. E. Glover, T. D. Donnelly, E. A. Lipman, A. Sullivan, and R. W. Falcone, Phys. Rev. Lett. 73, 78 (1994).

[27] S. C. Rae and K. Burnett, Phys. Rev. A 46, 2077 (1992).

[28] T. Ditmire, Ph.D. thesis, University of California, Davis, (1995), Lawrence Livermore National Laboratory Publication No. UCRL-123306.

[29] S. C. Wilks, W. L. Kruer, E. A. Williams, P. Amendt, and D. C. Eder, Phys. Plasmas 2, 274 (1995).

[30] J. K. Crane, H. Nguyen, T. Ditmire, C. Coverdale, T. E.
Glover, M. D. Perry, and Y. Zakharenkov, J. Opt. Soc. Am. B 13, 89 (1996).

[31] W. J. Blyth, S. G. Preston, A. A. Offenberger, M. H. Key, J. S. Wark, Z. Najmudin, A. Modena, A. Djaoui, and A. E. Dangor, Phys. Rev. Lett. 74, 554 (1995).

[32] W. Lotz, Z. Phys. 216, 241 (1968).

[33] L. D. Landau and E. M. Lifshitz, Electrodynamics of Continuous Media (Pergamon, Oxford, 1984).

[34] J. D. Jackson, Classical Electrodynamics (Wiley, New York, 1975).

[35] V. P. Silin, Zh. Eksp. Teor. Fiz. 47, 2254 (1964) [Sov. Phys. JETP 20, 1510 (1965)].

[36] A. F. Haught and D. H. Polk, Phys. Fluids 13, 2825 (1970).

[37] L. Spitzer, Physics of Fully Ionized Gases (Interscience, New York, 1967).

[38] M. M. Murnane, H. C. Kapteyn, and R. W. Falcone, Phys. Rev. Lett. 62, 155 (1989).

[39] T. E. Glover, J. K. Crane, M. D. Perry, R. W. Lee, and R. W. Falcone, Phys. Rev. Lett. 75, 445 (1995).

[40] R. C. Elton, in Plasma Physics, edited by H. R. Griem and R. H. Lovberg (Academic, New York, 1970).

[41] Y. B. Zel'dovich and Y. P. Raizer, in Physics of Shock Waves and High-Temperature Hydrodynamic Phenomena, edited by W. D. Hayes and R. F. Probstein (Academic, New York, 1966), Vol. 1.

[42] J. F. Luciani, P. Mora, and J. Virmont, Phys. Rev. Lett. 51, 1664 (1983).

[43] L. Spitzer and R. Härm, Phys. Rev. 89, 977 (1953).

[44] A. Djaoui and A. A. Offenberger, Phys. Rev. E 50, 4961 (1994).

[45] R. W. Lee (private communication).

[46] T. Ditmire, H. Nguyen, and M. D. Perry, J. Opt. Soc. Am. B 11, 580 (1994).

[47] T. Ditmire, H. Nguyen, and M. D. Perry, Opt. Lett. 20, 1142 (1995).

[48] M. D. Perry, C. Darrow, C. Coverdale, and J. K. Crane, Opt. Lett. 17, 523 (1992).

[49] O. Abraham, S.-S. Kim, and G. D. Stein, J. Chem. Phys. 75, 402 (1981).

[50] R. Klingelhöfer and H. O. Moser, J. Appl. Phys. 43, 4575 (1972).

[51] H. P. Birkhofer, H. Haberland, M. Winterer, and D. R. Worsnop, Ber. Bunsenges. Phys. Chem. 88, 207 (1984).

[52] B. J. C. Wu, P. P. Wegener, and G. D. Stein, J. Chem. Phys. 69, 1776 (1978)

[53] R. L. Kelly, Atomic and Ionic Spectrum Lines Below 2000 Angstroms: Hydrogen through Krypton (American Chemical Society, Washington, DC, 1987), Vol. 1.

[54] R. D. Bleach, J. Opt. Soc. Am. 70, 861 (1980).

[55] B. C. Fawcett and A. H. Gabriel, Proc. Phys. Soc. London 84, 1038 (1964).

[56] B. C. Fawcett, A. H. Gabriel, B. B. Jones, and N. J. Peacock, Proc. Phys. Soc. London 84, 257 (1964).

[57] M. Mohan, M. L. Dourneuf, and A. Hibbert, J. Phys. B 24, 299 (1991).

[58] T. Ditmire, J. K. Crane, H. Nguyen, L. B. DaSilva, and M. D. Perry, Phys. Rev. A 51, R902 (1995). 